\title{
Probabilités d'occurrence des séquences sèches les plus longues sur le bassin versant du Gardon (sud-est de la France) - Quels impacts de la définition du jour sec sur les distributions statistiques des séquences sèches les plus longues en climat méditerranéen?
}

\author{
Hadrien Di Costanzo * \\ Avignon université, UMR ESPACE 7300 du CNRS \\ 74, rue L. Pasteur, 84029 Avignon cedex - France
}

\begin{abstract}
Résumé - En climat méditerranéen, l'évapotranspiration potentielle est forte, en particulier durant la saison estivale. Elle coïncide avec une période de faible pluviosité. Si celle-ci perdure durant des phases de forte évapotranspiration, elle peut avoir de graves conséquences sur les activités agricoles très consommatrices en eau pendant leur période de croissance. Une étude de la sécheresse atmosphérique dans le secteur du Gardon est réalisée à partir de l'indicateur des séquences sèches. Premièrement, une étude historique permet d'identifier les grands évènements de sécheresse dans le bassin du Gardon entre 1900 et 2019. Puis une analyse sur l'effet du seuil de pluie pour caractériser les précipitations utiles est proposée. Différentes définitions du jour sec sont testées pour calculer les séquences sèches entre 2000 et 2019. L'utilisation d'un seuil d'évapotranspiration réelle ou d'évapotranspiration potentielle, par rapport à un seuil de $1 \mathrm{~mm}$, modifie à probabilité d'occurrence égale la saisonnalité et la géographie de l'intensité des séquences sèches. Les seuils d'évapotranspiration réelle et d'évapotranspiration potentielle produisent des évènements plus intenses qu'un seuil de $1 \mathrm{~mm}$. L'intensité des séquences sèches est sous-estimée avec un seuil de $1 \mathrm{~mm}$ pour les évènements des mois de printemps mais surtout pour ceux de début d'été. Sont identifiés à risque, quel que soit le seuil retenu, les secteurs du sous-bassin de la Salindrenque et la partie la plus aval du bassin du Gardon. Elles sont touchées à probabilité d'occurrence égale par des intensités plus importantes pour les évènements de moyenne et de forte intensité que le reste du bassin. La comparaison des distributions statistiques en fonction du seuil retenu montre que, à défaut d'avoir une valeur d'évapotranspiration réelle pour déterminer les précipitations utiles, le seuil $1 \mathrm{~mm}$ reste plus pertinent qu'un seuil d'évapotranspiration potentiel.
\end{abstract}

Mots-clés : Climat méditerranéen, séquences sèches, évènements climatiques extrêmes, évapotranspiration, bassin versant du Gardon.

\begin{abstract}
Probabilities of occurrence of the longest dry spells in the Gardon watershed (southeastern France) - What are the impacts of the definition of the dry day on the statistical distributions of the longest dry spells in Mediterranean climate? In the Mediterranean climate, potential evapotranspiration is high, especially during the summer season. It coincides with a period of low rainfall. If this period persists during phases of high evapotranspiration, it can have serious consequences for agricultural activities that consume a lot of water during their growth period. First, a study of atmospheric drought in the Gardon sector is conducted with the indicator of dry sequences. Then, the historical analysis allows the identification of major drought events in the Gardon basin between 1900 and 2019. An analysis of the effect of the rainfall threshold is proposed to characterise the effective rainfall. Different definitions of the dry day are tested to calculate the dry sequences between 2000 and 2019. The use of an actual evapotranspiration or potential evapotranspiration threshold, as compared to a $1 \mathrm{~mm}$ threshold, changes the seasonality and geography of the intensity of dry sequences given equal probability of occurrence. Both real and potential evapotranspiration thresholds produce more intense events than a $1 \mathrm{~mm}$ threshold. The intensity of the dry sequences is underestimated with a threshold of $1 \mathrm{~mm}$ for the events of the spring months but especially for the events of the beginning of the summer. The sectors identified as potentially at risk, regardless of the threshold used, are the Salindrenque sub-basin and the most
\end{abstract}

This is an Open Access article distributed under the terms of the Creative Commons Attribution License CC-BY-NC (https://creativecommons.org/licenses/by-nc/4.0), which permits unrestricted use, distribution, and reproduction in any medium, except for commercial purposes, provided the original work is properly cited. 
downstream part of the Gardon basin. They are affected, with equal probability of occurrence, by greater intensities for medium and high intensity events than the other parts of the basin. The comparison of the statistical distributions according to the selected threshold shows that, in the absence of a real evapotranspiration value to determine the effective precipitation, the $1 \mathrm{~mm}$ threshold remains more relevant than a potential evapotranspiration threshold.

Keywords: Mediterranean climate, dry spells, extreme climatic event, evapotranspiration, Gardon Watershed.

* $\overline{\text { Auteur de correspondance }}$ : hadrien.di-costanzo@univ-avignon.fr

\section{Introduction}

On distingue habituellement quatre types de sécheresse (Dracup et al., 1980 ; Wilhite et Glantz, 1985 ; Mishra et Singh, 2010 ; Van Loon et al., 2016) : atmosphérique, édaphique, hydrologique et sociétale. Les trois premières traduisent un déficit hydrique dans différents réservoirs du cycle hydrologique. Ces déficits impactent in fine le bon fonctionnement d'une société : pertes agricoles, feux de forêt, dégradations des écosystèmes, baisse de la production énergétique, etc. (Aguilera-Klink et al., 2000 ; Wilhite et al., 2007 ; Van Loon et al., 2016a ; Van Loon et al., 2016b ; Guermazi et al., 2019). Contrairement aux crues, il s'agit d'un aléa à cinétique lente (mois ou années) agissant sur de vastes espaces géographiques (bassin versant, pays ou région), ce qui complique la gestion de crise (Karavitis et al., 2014 ; Van Loon, 2015). La sécheresse atmosphérique correspond à une longue période d'absence de pluie pouvant être associée à des conditions de forte évapotranspiration (Senaut, 2015). Elle est à l'origine de toute sécheresse.

La sécheresse est récurrente en Méditerranée. La remontée vers le nord des anticyclones subtropicaux durant l'été décale les retombés d'air de la cellule de Hadley au niveau du pourtour méditerranéen et implique une diminution des précipitations. Elle coïncide avec la période la plus chaude de l'année. Cette situation est propice à la génération de sécheresse atmosphérique.

Ce secteur géographique est considéré par Giorgi (2006) comme un hotspot du changement climatique. De nombreux travaux ont montré une diminution récente des précipitations, accompagnée d'une augmentation des températures en secteur méditerranéen (Norrant et Douguédroit, 2006 ; Sousa et al., 2011 ; Milano et al., 2013 ; Spinoni et al., 2017 ; Stagge et al., 2017). Une possible aggravation de ces tendances est à envisager d'ici la fin du XXI ${ }^{\mathrm{e}}$ siècle (Arnell, 2003 ; Giorgi, 2006; Van der Schrier et al., 2006 ; Burke et Brown, 2008 ; de Marsily, 2010 ; Dai, 2011 ; Milano et al., 2013 ; Vicente-Serrano et al., 2014 ; Tramblay et al., 2020 ; Vicente-Serrano et al., 2020). Par ailleurs, plusieurs études ont confirmé un assèchement du territoire français, en particulier dans l'espace méditerranéen. Le projet ClimSec (Soubeyroux et al., 2011) souligne une augmentation de l'intensité et de la longueur des sécheresses édaphiques durant la seconde moitié du $\mathrm{XX}^{\mathrm{e}}$ siècle. D'autres études, plus locales, constatent également une légère baisse des précipitations dans le sud-est de la France (Norrant et Douguédroit, 2004) et sur certains petits bassins versants méditerranéens français (Folton $e t$ al., 2020). Dans un contexte de changement climatique (diminution des précipitations associée à une augmentation de l'évapotranspiration) et de renforcement de la pression anthropique sur les bassins versants (modes d'irrigations peu économes, cultures non adaptées au climat local, augmentation de la population accentuée par une forte affluence touristique), une diminution de la ressource en eau est à craindre à l'avenir.

Il est proposé dans cette étude une analyse de la sécheresse atmosphérique sur un bassin versant méditerranéen du sud-est de la France : le Gardon. Après avoir présenté les données et la méthodologie suivie, nous exposerons dans un premier temps une analyse historique entre 1900 et 2019 de la variation des sécheresses atmosphériques les plus intenses à partir des données pluviométriques des stations Météo France. Puis dans un second temps, nous proposons sur la période 2000-2019, toujours dans le périmètre du bassin du Gardon, une analyse critique sur le choix de la définition du jour sec. Ce biais est souvent mis de côté pour le calcul des séquences sèches (indicateur retenu pour étudier les sécheresses atmosphériques). Nous verrons qu'il peut amener à reconsidérer la probabilité 
d'occurrence des évènements extrêmes ainsi que leurs distributions spatiales sur le bassin.

\section{Données}

\subsection{Terrain d'étude}

Le bassin du Gardon (figure 1) est un sous-bassin du Rhône d'environ 2000 km². Dans ce secteur, les phénomènes de sécheresse sont moins étudiés que les crues cévenoles. Or, les enjeux sur la ressource en eau sont importants, tout particulièrement en période estivale où l'on retrouve de fortes tensions entre les usages touristiques et agricoles qui sont des secteurs stratégiques pour l'économie du département du Gard. La surface agricole utile représente $42 \%$ de la superficie du bassin versant avec la vigne pour culture dominante (www.agreste.agriculture.gouv.fr/agresteweb/disaron/Carte-RA-sauev/detail/). La partie aval du bassin est la plus cultivée. On y retrouve en particulier des cultures fruitières, maraîchères et de la viticulture. En amont du bassin, sur la partie cévenole, l'agriculture est dite traditionnelle et extensive avec une activité d'élevage prédominante.
En période estivale, la consommation d'eau par les agriculteurs représente une part majoritaire de la consommation totale sur ce bassin. D'après le plan de gestion de la ressource en eau du bassin du Gardon (Établissement Public Territorial de Bassin Gardons, 2018), le prélèvement net pour l'irrigation est estimé à $1,2 \mathrm{Mm}^{3}$ durant le mois de juin (mois de pointe du prélèvement). L'irrigation des cultures est assurée par plusieurs systèmes d'irrigation. Le canal de Boucoiron, de Beaucaire et les périmètres d'irrigation gérés par la société BRL contribuent principalement à l'irrigation des cultures dans la partie aval du bassin. Sur la partie amont, l'irrigation est gérée de manière plus informelle à partir des eaux de rivières détournées par de nombreux béaux (petits canaux de dérivation d'eau dans le sud-est de la France). En cas d'arrêtés préfectoraux, le prélèvement peut être limité puis interdit (Riaux et al., 2009). Les conséquences sur le rendement peuvent être très sévères en période de croissance végétative dans un secteur où l'agriculture est fortement dépendante de l'irrigation en période estivale.

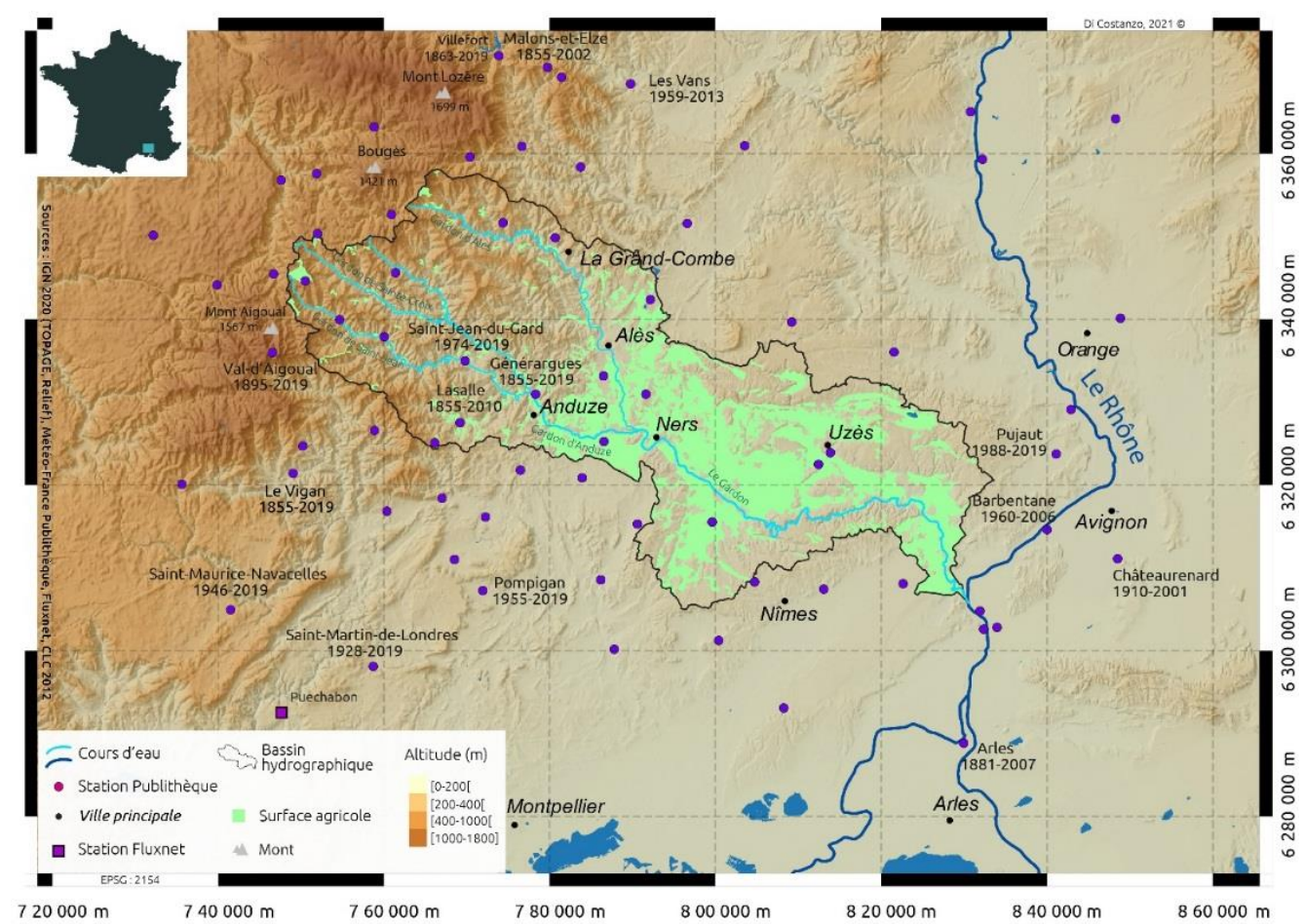

Figure 1. Carte du secteur du bassin versant du Gardon ; 59 stations météorologiques disponibles sur plus de 30 ans sont recensées entre 1855 et 2019, et 44 entre 2000 et 2019. Les stations utilisées pour l'observation séculaire des sécheresses atmosphériques sont identifiées et leur fenêtre temporelle de mesure précisée sur la carte. Map of the Gardon watershed area. 59 meteorological stations available over 30 years are identified between 1855 and 2019 and 44 between 2000 and 2019. The stations used for the secular observation of atmospheric droughts are identified and their period measurement is specified on the map. 


\subsection{Les données satellites MODIS}

L'ETR est couramment estimée à partir de la méthode de Thornthwaite (1948). Elle est simple à calculer, mais conduit à faire des hypothèses fausses sur les caractéristiques de la réserve utile. Depuis quelques décennies, plusieurs satellites proposent une mesure spatiale et temporelle de l'ETR. Zhang et al. (2016) présentent une revue détaillée de l'historique des méthodes mises en œuvre pour l'estimation de l'ETR par satellite. Elles reposent sur la résolution du bilan d'énergie qui fournit le lien explicite entre la température mesurée et le flux de chaleur sensible et latente. Il faut cependant rappeler que ces données radiométriques satellitaires sont impactées par une dégradation de la qualité de la mesure en présence d'une quantité importante d'aérosols dans l'atmosphère ou lors de la présence de nuages (cause principale des biais). Les séquences sèches les plus longues surviennent généralement en période de ciel dégagé, sans pluie. On peut faire l'hypothèse que ce biais métrologique impacte moins le calcul des séquences sèches les plus longues dans le sud-est de la France. Les données satellites MODIS ont été choisies en fonction de leur résolution temporelle et spatiale. Le produit MOD16A2 (Running et al., 2019) donne une estimation de l'ETR et de l'ETP à partir du modèle de Penman-Monteith (Monteith, 1965). La donnée est produite à une résolution de $500 \mathrm{~m}$ sous la forme d'un cumul hebdomadaire depuis 2000.

Pour limiter l'impact de certaines valeurs aberrantes sur notre analyse, nous avons (1) retiré les pixels identifiés comme défectueux par le producteur, (2) moyenné la valeur des pixels dans un périmètre d' $1 \mathrm{~km}^{2}$ avoisinant la station afin de s'affranchir d'éventuels cas de pixels défectueux (population de neuf pixels voisins) et enfin, (3) interpolé temporellement par la méthode dite LOWESS (Cleveland, 1981) les séries chronologiques hebdomadaires des moyennes spatiales des cumuls d'ETP et d'ETR afin de réduire le bruit et estimer les valeurs quotidiennes (Moreno et al., 2014). A partir de 2000, la mise en service relativement récente du satellite MODIS permet la comparaison des distributions des séquences sèches (seuil de $1 \mathrm{~mm}$, ETP, ETR) (figure 2a).

\subsection{Les postes météorologiques Météo France}

Les postes de Météo France recensés par la
Publithèque (publitheque.meteo.fr/okapi/accueil/) sont utilisés pour les mesures de précipitation et d'ETP. Nous retenons l'ensemble des postes pluviométriques comportant plus de 30 années de mesures situées dans le bassin versant du Gardon et à sa proximité. L'emprise géographique est comprise entre $3,1^{\circ} \mathrm{E}$ et $6,2^{\circ} \mathrm{E}$, et entre $43,0^{\circ} \mathrm{N}$ et $44,7^{\circ} \mathrm{N}$ (figure 1 ).

Plusieurs chroniques permettent une analyse séculaire, la plus longue étant la chronique de Générargue mesurant les hauteurs de précipitation depuis 1855. Excepté la période antérieure à 1900, le taux d'absence de données par poste chaque année reste très faible (moins de 5 absences quotidiennes/poste/an) (figure 2a). On distingue, pour l'évolution du nombre de postes trois grandes périodes principalement liées à l'histoire économique du pays :

1) une période antérieure à 1950 durant laquelle le nombre de postes est compris entre 10 et 20 ;

2) une période après la Seconde Guerre Mondiale avec une augmentation rapide du nombre de stations (de 20 à 43) jusqu'en 1970 ;

3 ) enfin, une stagnation du nombre de postes autour de 45 entre 1970 et 2019.

Le nombre de postes mesurant le cumul quotidien de précipitation sur la période 1855 à 1945 (entre 1 et 20 postes) est moins important qu'à la fin (une quarantaine). La représentativité spatiale des mesures pluviométriques au cours du temps peut être remise en cause (figure 2a). Le faible nombre de postes et la part importante de valeurs absentes nous ont conduits à retirer les mesures antérieures à 1900 (figure 2a).

Lorsqu'elles sont disponibles pour ces postes pluviométriques, nous récupérons également les mesures d'ETP (figure 2a). Le nombre de postes mesurant l'ETP et la couverture temporelle associée sont beaucoup moins grands que pour la pluviométrie (4 postes sur 44 entre 2000 et 2019). Plusieurs types de méthodes d'estimation de l'ETP existent (Lecarpentier, 1975) : thermiques (Hargreaves), énergétiques (Priestley-Taylor) et de transfert de masse (Dalton). Dans notre cas, l'estimation est faite à partir de la méthode Penman-Monteith modifiée par Météo France (Ducharne, 2002). Vannier et Braud (2012) ont comparé différentes variantes de la formule de calcul de l'ETP Penman-Monteith, dont 
celle utilisée par Météo France dans la Publithèque dans le département du Gard. Les mesures ont été jugées satisfaisantes, car concordantes avec d'autres méthodes de calcul de l'ETP. Elles sont donc mobilisées pour valider les estimations d'ETP par satellite.
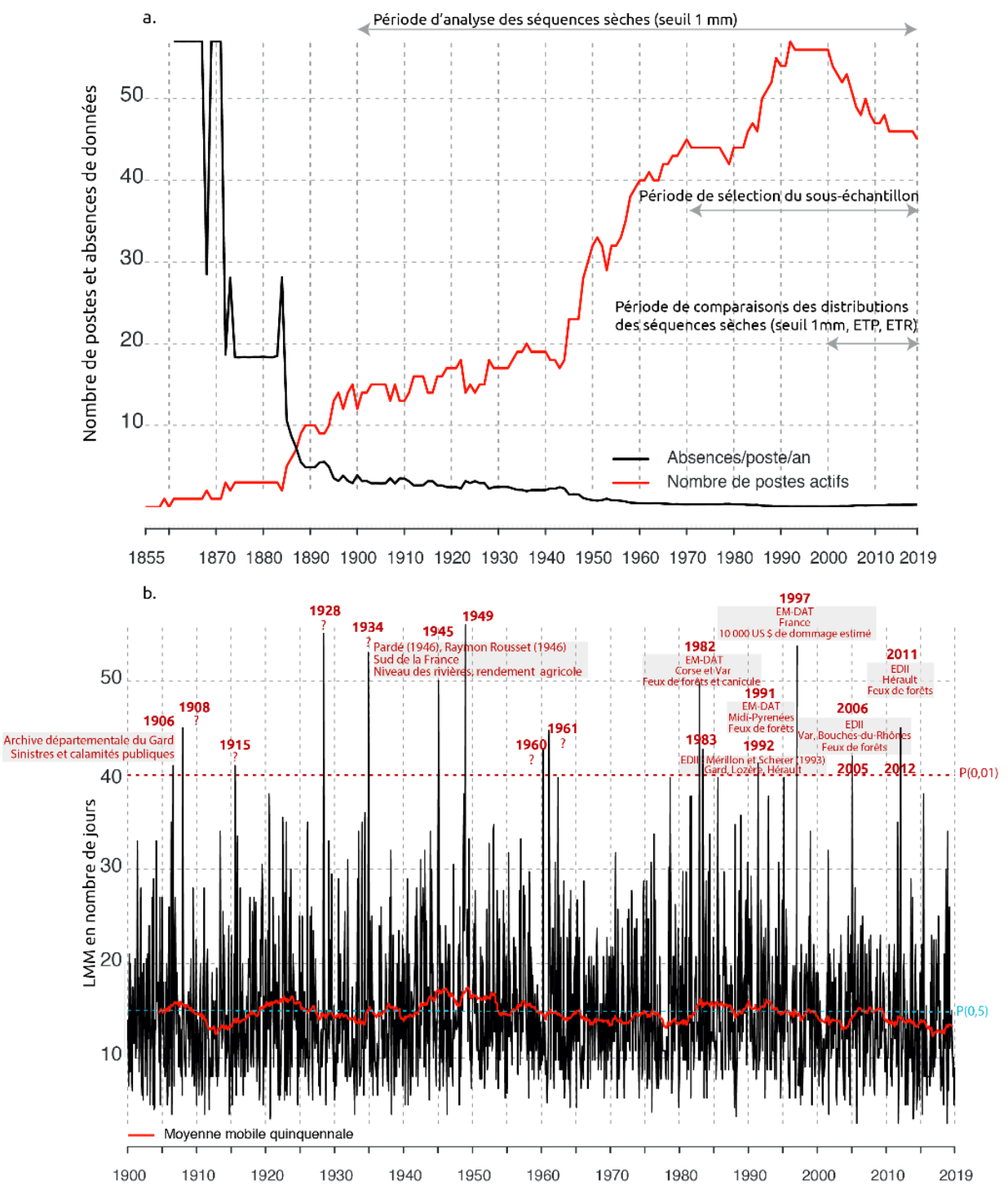

Figure 2. a) Nombre de postes disponibles et taux de lacune (nombre d'absences quotidiennes/poste/an) des pluviomètres Météo France de la Publithèque mesurant plus de trente années de mesure sur le secteur du bassin du Gardon (1855-2019). b) Variabilité temporelle des sécheresses atmosphériques mesurées à partir des longueurs maximales mensuelles (LMM) des séquences sèches (seuil de $1 \mathrm{~mm}$ ) calculées sur les postes pluviométriques entre 1855 et 2019. La moyenne d'un sous échantillon représentatif du secteur déterminé à partir de tests de Monte-Carlo a été utilisée pour décrire l'évolution des évènements de sécheresse atmosphérique sur le secteur du bassin du Gardon entre 1900 et 2019. Les années précisées sur le graphique sont celles ayant une probabilité d'occurrence inférieure ou égale à 0,01 . Lorsque des impacts de sécheresses ont été identifiés par d'autres sources, les références, le lieu et le type d'impact observé sont précisés. Les points interrogation signalent une absence de source pour la confirmation de l'évènement à partir d'impacts observés. a) Number of available stations and gap rate (number of daily absences/station/year) of the Publithèque Météo France rain gauges measuring more than 30 years over the Gardon basin area (1855-2019). b) Temporal variability of atmospheric droughts measured from the maximum monthly lengths (MML) of dry sequences (1 $\mathrm{mm}$ threshold) calculated on rain gauge stations between 1855 and 2019. The average of a representative subsample of the sector determined from Monte-Carlo tests was used to describe the evolution of atmospheric drought events over the Gardon basin sector between 1900 and 2019. The years specified on the graph represent events with a probability of occurrence inferior or equal to 0.01 . When drought impacts have been identified by other sources, the references, location and type of impact observed are specified. Question marks indicate a lack of sources to confirm the event from observation impacts. 


\subsection{La tour de flux de turbulence du réseau Fluxnet}

Les données de la station de flux de turbulences Puechabon du réseau Fluxnet (Pastorello, 2020) ont été utilisées pour vérifier la qualité d'estimation de l'ETR MODIS par rapport aux mesures in situ. Les stations de tours de flux de turbulence sont utilisées pour mesurer l'ETR d'un couvert forestier de quelques kilomètres carrés. Elles sont donc compatibles avec la résolution du satellite $(500 \mathrm{~m})$. Les mesures d'ETR de la station de flux de turbulences sont disponibles entre 2003 et 2014 à une échelle horaire (sites.fluxdata.org/FR-Pue/) (figure 2a). Les cumuls hebdomadaires sont calculés pour rendre la comparaison possible avec les données MODIS. La station de Puechabon est proche du secteur. Elle est située au sud-ouest du bassin versant du Gardon à une cinquantaine de kilomètres (figure 1) et représentative d'une végétation de type méditerranéenne.

\section{Méthodes}

\subsection{Calcul des séquences sèches et de leur intensité}

Différents indicateurs sont utilisés pour l'observation de la sécheresse atmosphérique : par exemple celui appelé Standardised Precipitation Index (McKee et al., 1993) ou le Palmer Drougth Severity Index (Palmer, 1965). L'utilisation des cumuls ou moyennes mensuelles de précipitations ne permet pas de caractériser la structure temporelle des jours sans pluie. Or, elle est aussi déterminante que la quantité d'eau précipitée dans des secteurs où les précipitations sont nombreuses et intenses. Nous utiliserons dans cette étude l'indicateur des séquences sèches initialement proposé par Douguédroit (1980), adapté dans le sud-est de la France.

Une séquence sèche est définie comme une succession ininterrompue de jours secs (Douguédroit, 1980 ; Peiris, 2011). Afin de limiter les erreurs de calcul, la première et la dernière séquence sèche de chaque série temporelle de mesure du cumul quotidien de précipitation ont été éliminées. Il en est de même pour les séquences qui encadrent une valeur absente de cumul quotidien de pluie. Parmi les différentes études menées sur cet indicateur, plusieurs mesures de l'intensité ont été proposées. Des auteurs ont travaillé sur la longueur maximale annuelle (Caloiero et al., 2015 ; Tramblay et Herting, 2018), la longueur moyenne annuelle (Douguédroit, 1980 ; Douguédroit, 1983 ; Douguédroit 1987), les longueurs supérieures à un certain seuil de percentile (Lana et al., 2006 ; Lana et al., 2008 ; Caloiero et al., 2015 ; Raymond et al., 2016), le nombre de jours secs (Douguédroit, 1983 ; Raymond et al., 2016), à partir des périodes de retour (Douguédroit, 1990 ; Lana et al., 2006). Nous faisons le choix de travailler sur la longueur des séquences sèches maximales mensuelles (LMM). Celles-ci permettent d'avoir un sous-échantillon des extrêmes plus robustes qu'un maximum annuel (12 LMM/an). De plus, chaque mois de l'année est considéré et une analyse saisonnière devient possible à partir de ce sous-échantillonnage. Chaque LMM est déterminée à partir de la date de départ de la séquence sèche. La longueur de la séquence sèche est utilisée pour mesurer l'intensité d'une sécheresse atmosphérique. La probabilité d'occurrence permet de comparer, à intensité égale, le risque d'apparition d'une séquence sèche entre différents secteurs géographiques, périodes de l'année ou méthodes de calcul.

\subsection{Choix du seuil du jour sec}

Dans le cadre des études sur les séquences sèches, le jour sec est couramment défini par un jour sans pluie ou encore par un jour avec des précipitations inférieures à $1 \mathrm{~mm}$ pour prendre en compte l'évaporation ou l'erreur de mesure (Peiris et al., 2011). Ce choix de seuil ne nous semble pas pertinent en secteur méditerranéen. Quelques auteurs ont travaillé sur la distribution statistique des séquences sèches à partir de différents seuils de précipitation (Lana et al., 2008). Ceux-ci ont montré que le choix du seuil ne change pas fondamentalement la structure de la distribution statistique. Néanmoins, l'utilisation d'un seuil fixe tout au long de l'année ne prend pas en compte l'effet du cycle annuel de l'évapotranspiration.

Rivoire et al. (2019) ont pointé sur le pourtour méditerranéen des différences notoires pour les tendances et la forme des distributions statistiques des séquences sèches les plus intenses en comparant l'effet d'un seuil fondé sur l'évapotranspiration potentielle (ETP) avec celui d'1 mm. Si l'ETP 
permet de mieux prendre en compte la saisonnalité de l'évapotranspiration et donc de préciser la mesure des précipitations utiles (PU), on peut suspecter dans un bassin versant une surestimation de la quantité d'eau évaporée. Le contrôle végétatif de l'évaporation et la taille de la réserve utile sont rarement négligeables. Au cours de la période estivale, la disponibilité en eau dans les sols limite l'évapotranspiration dans le sud de la France.

Nous proposons l'utilisation de trois seuils pour déterminer les PU : $1 \mathrm{~mm}$, l'ETP et l'ETR. L'ETP défini par Thornthwaite (1948) correspond à la quantité d'eau évaporée et transpirée par le sol dans une situation théorique où l'alimentation en eau serait illimitée (Mounier, 1977 ; Carrega, 1988). L'évapotranspiration réelle (ETR) exprime la quantité d'eau réellement évapotranspirée. Elle est dépendante de l'ETP (la demande atmosphérique) et du niveau de la réserve utile (l'offre) (Mounier 1977 ; Carrega, 1988). Nous proposons de comparer les distributions des LMM calculées en fonction de ces différents seuils.

\subsection{Détermination des lois de distribution}

Initialement, les chaînes de Markov ont été utilisées pour déterminer la fonction de probabilité des séquences sèches (Garcia et Martin-Vide, 1993). Mais, celles-ci induisent des biais d'estimation de la probabilité d'occurrence importants pour les séquences sèches les plus courtes et les plus longues (Douguedroit, 1983 ; Peiris, 2011). Plusieurs lois de distribution ont donc été introduites pour la détermination de la fonction de probabilité d'occurrence de l'intensité des séquences sèches : binomiale négative tronquée (Douguedroit, 1990), Poisson (Lana et Burgueno, 1998), logarithmique (Kamar et Rao, 2004), Weibull (Lana et al., 2008), la famille des distributions géométriques de séries logarithmiques mixtes (Deni et Jemain, 2009), la famille des valeurs extrêmes généralisées (Lana et al., 2006 ; Rivoire et al., 2019), Log-Normale (Rivoire et al., 2019), Gumbel (Perzyna, 1994 ; Vicente-Seranno et Begueria, 2003 ; Lana et al., 2006), Generalised Pareto (Lana et al., 2006). Leurs utilisations varient en fonction de l'aire géographique et de la méthode de mesure de l'intensité.

Le package GAMLSS (Generalized Additive
Models for Location, Scale and Shape) (Stasinopoulos et Rigby, 2007 ; Rigby et al., 2017) du logiciel R (www.R-project.org/) est utilisé pour déterminer les lois et paramètres les plus adéquats. Ce package permet, à partir des critères d'information bayésien et d'information d'Akaike (indicateurs de qualité d'un modèle à partir de sa parcimonie et de sa vraisemblance), de proposer des lois adaptées aux distributions statistiques étudiées. L'ensemble des lois citées précédemment sont testées.

\subsection{Choix de l'échantillon spatial et temporel représentatif des LMM du bassin du Gardon}

Des tests de Monte-Carlo (1000 tirages aléatoires d'un sous-échantillon de 15 stations) sur la période durant laquelle le nombre de postes est le plus important (1970 à 2019) sont réalisés. Pour chaque année, les moyennes spatiales de ces 1000 tirages aléatoires sont comparées à la moyenne spatiale de l'ensemble des stations disponibles. Parmi ces 1000 tirages, le groupe de stations dont les écarts chaque année entre 1970 et 2019 à la moyenne spatiale étaient les plus faibles et la couverture temporelle la plus longue est retenu pour décrire l'évolution des LMM entre 1900 et 2019. Nous faisons l'hypothèse que l'homogénéité observée à partir de ce sous-échantillonnage sur 50 ans reste représentative au cours de la période antérieure. Cette hypothèse pourrait être acceptable si les séries retenues sont stationnaires.

\subsection{Les tests statistiques utilisés}

Les tendances séculaires sont contrôlées à partir du test non paramétrique de Man-Kendall (Mann, 1945 ; Kendall, 1990) avec un seuil de significativité de 0,05 . Il présente l'avantage d'être moins sensible aux valeurs singulières et n'est pas affecté par la distribution des données. Le test est calculé à partir du package Rtrend (cran.rproject.org/web/packages/rtrend/index.html) du logiciel R. Les écarts de valeurs entre distributions sont testés à partir d'un test de Wilcoxon (Wilcoxon, 1947) pour comparer des différences entre des distributions qui ne sont pas stricto sensu normales mais dont la symétrie est respectée. Le test de Shapiro-Wilk (Shapiro et Wilk, 1965) est utilisé pour contrôler la normalité des résidus d'ajustements de lois statistiques. 


\section{Résultats}

\subsection{Sélection des séries chronologiques des LMM représentatives du bassin du Gardon}

\subsubsection{Les précipitations (1900-2019)}

Les tests de Monte-Carlo permettent d'identifier 15 stations représentatives du secteur entre 1900 et 2019. Ce sous-échantillonnage présente entre 1970 et 2019 une valeur moyenne associée à un intervalle de confiance $(\mathrm{P}<0,05)$ de 14,98 $\pm 1,97$ jours très similaires à celle de l'ensemble de l'échantillon $(14,94 \pm 4,57$ jours $)$ et des 1000 tirages aléatoires des 15 stations $(15,30 \pm 7,20$ jours). Ces résultats confirment l'homogénéité spatiale et temporelle d'un sous-échantillonnage dans le secteur, bien qu'en moyenne l'utilisation d'un jeu de 15 stations ait tendance à surestimer les LMM du secteur. Aucune tendance significative n'a été détectée dans le sous-échantillon de 15 stations retenues (tableau 1). Une représentativité entre 1900 et 1970 nous semble acceptable. La moyenne des stations suivantes est retenue : Le Vigan (1855 à 2019), Générargues (1855 à 2019), Villefort (1863 à 2019), Lasalle (1855 à 2010), Malons-et-Elze (1855 à 2002), Arles (1881 à 2007), Val d'Aigoual (1895 à 2019), Saint-Martin-de-Londres (1928 à 2019), SaintMaurice-Navacelles (1946 à 2019), Pompignan (1955 à 2019), Les Vans (1959 à 2013), Barbentane (1960 à 2006), Châteaurenard (1910 à 2001), Saint-Jean-duGard (1974 à 2019), Pujaut (1988 à 2019) (figure 1).

\subsubsection{L'ETP des données MODIS et de la Publithèque (2000-2019)}

La relation statistique entre les mesures de

Tableau 1. Résultats du test de Mann et Kendall sur la moyenne du sous-échantillon des LMM (1900 à 2019) retenue pour le bassin versant du Gardon ; avec S la pente de Sen dont le signe renseigne sur le sens de la pente et la valeur sa force ; P, la P-value du test. Results of the Mann and Kendall test on the means of LMM (from 1900 to 2019) subsample for the Gardon Watershed; with $S$ the slope of Sen where the sign indicates the direction of the slope and the value its strength and $P$, the $P$-value of the test.
Penman-Monteith in situ et celles du satellite MODIS (figure $3 \mathrm{a}$ ) donne un $\mathrm{R}^{2}$ de $0,87(\mathrm{P}<0,05)$ ; $95 \%$ des résidus montrent des différences de mesures comprises entre $-9,1$ et $9,8 \mathrm{~mm} /$ semaine. On constate une surestimation de l'ETP par le satellite MODIS pour les valeurs les plus élevées (supérieures à $56 \mathrm{~mm} / \mathrm{semaine}$ ). La linéarité de la relation confirme que les deux séries temporelles d'estimation de l'ETP sont en phase. Bien qu'il existe des différences entre les deux approches d'estimation (4,69 $\mathrm{mm} /$ semaine en moyenne), elles restent cohérentes.

\subsubsection{L'ETR des données MODIS et de la station Puechabon du réseau Fluxnet (2000-2019)}

Avec l'ETR, on observe un $\mathrm{R}^{2}$ de 0,65 ( $\left.\mathrm{P}<0,05\right)$ entre les mesures satellites et celles in situ (figure 3b) ; $95 \%$ des résidus expriment une différence de mesures comprise entre $-0,9$ et $1 \mathrm{~mm} / \mathrm{semaine}$. On distingue des différences plus élevées pour les valeurs les plus faibles (entre 0 et $5 \mathrm{~mm} / \mathrm{semaine}$ ) et les plus fortes (supérieur à $25 \mathrm{~mm} / \mathrm{semaine}$ ). Les mesures MODIS descendent rarement en dessous d'une valeur d'environ $6 \mathrm{~mm} / \mathrm{semaine}$ pour les minimas alors que les mesures in situ peuvent être très proches de zéro en hiver. Les pics d'ETR de fin de printemps ou de début d'été sont beaucoup plus marqués sur les mesures in situ. La linéarité de la relation montre qu'il n'y a pas de déphasage temporel entre les deux chroniques. Bien qu'il existe des différences entre les deux méthodes $(1,3 \mathrm{~mm} / \mathrm{semaine}$ en moyenne), ces mesures restent cohérentes du point de vue de la variation saisonnière.

\begin{tabular}{|c|c|c|}
\hline Périodes & S & P \\
\hline Tous mois confondus & -1758 & 0,24 \\
\hline Janvier & -804 & 0,06 \\
\hline Février & 121 & 0,78 \\
\hline Mars & 200 & 0,65 \\
\hline Avril & -128 & 0,77 \\
\hline Mai & -204 & 0,64 \\
\hline Juin & 451 & 0,30 \\
\hline Juillet & -501 & 0,25 \\
\hline Août & -242 & 0,58 \\
\hline Septembre & -271 & 0,53 \\
\hline Octobre & -42 & 0,92 \\
\hline Novembre & 21 & 0,96 \\
\hline Décembre & -359 & 0,41 \\
\hline \multicolumn{2}{|c|}{}
\end{tabular}




\section{2. Évolution temporelle des LMM (seuil 1 $\mathrm{mm}$ ) sur le secteur du bassin versant $\mathrm{du}$ Gardon (1900-2019)}

\subsubsection{Les évènements historiques majeurs}

On ne distingue pas d'évolution des LMM entre 1900 et 2019. La chronique de la moyenne des LMM du sous-échantillon séculaire présente une tendance à la diminution non significative (tableau 1). Les tendances de chaque mois sont également non significatives. Concernant les évènements de très forte intensité, c'est-à-dire ayant une probabilité d'occurrence inférieure ou égale à 0,01 (40 jours), le record est détenu par l'année 1949 avec 56 jours secs consécutifs sur le secteur étudié.

Figure 3. a) Relation statistique sur la période 2000-2019 entre les estimations in situ d'ETP des cumuls hebdomadaires des stations Nîmes, Orange, Grande Combe, Pujaut et les cumuls hebdomadaires d'ETP du satellite MODIS. b) Relation statistique sur la période 2003-2014 entre les estimations in situ d'ETR des cumuls hebdomadaires de la station Puechabon du réseau Fluxnet et les cumuls hebdomadaires d'ETR du satellite MODIS. a) Statistical relationship over the period 2000-2019 between the in situ ETP estimates of the weekly totals of the stations Nîmes, Orange, Grande Combe, Pujaut and the weekly ETP totals of the MODIS satellite. b) Statistical relationship over the period 2003-2014 between the in situ ETP estimates of the weekly totals of the station Puechabon of the Fluxnet network and the weekly ETP totals of the MODIS satellite.
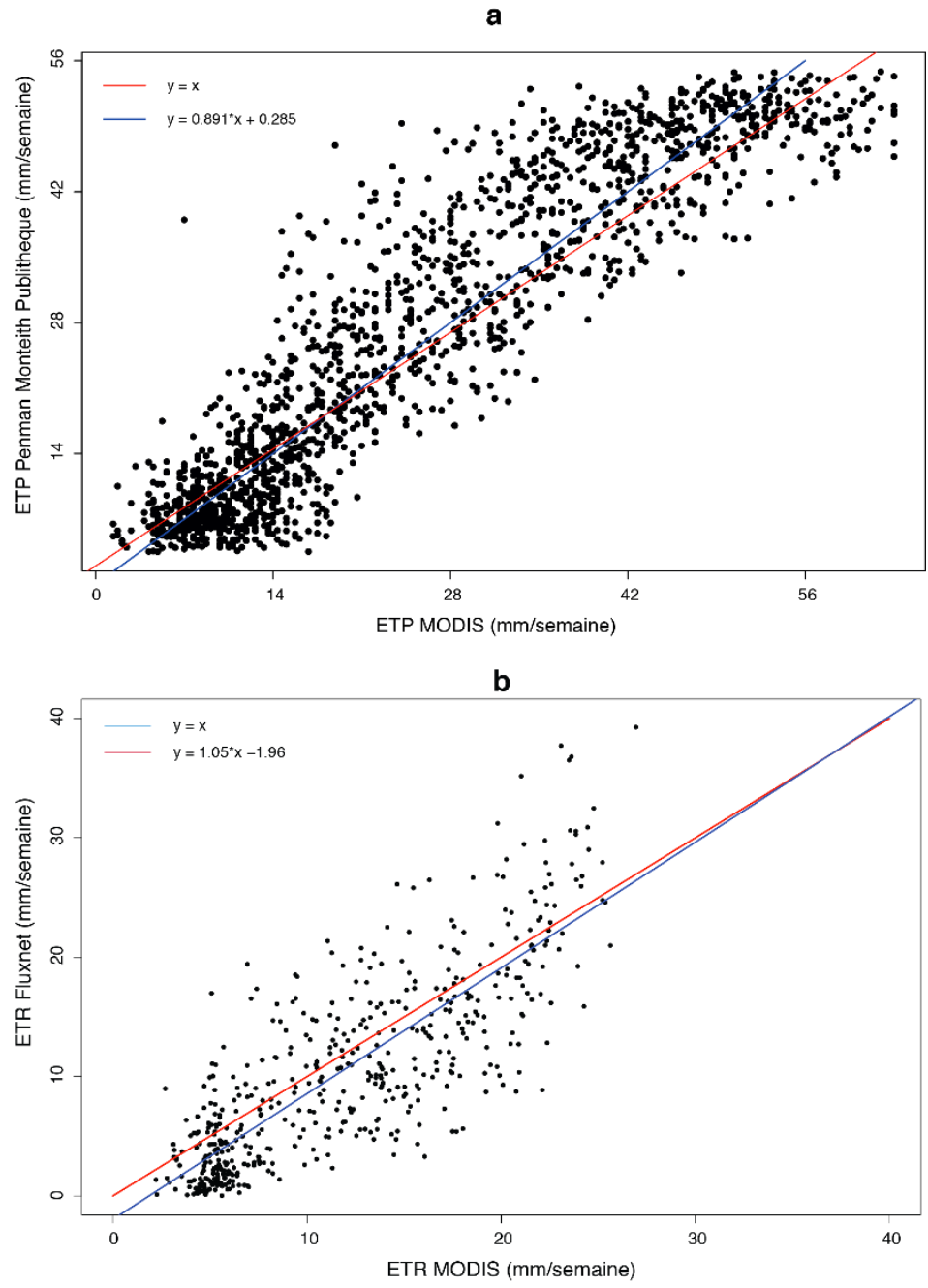

Dans l'ordre décroissant d'intensité, on retient pour les 14 autres années : 1928 (55 jours), 1934 (53 jours), 1997 (53 jours), 1945 (50 jours), 1982 (49 jours), 1908 (45 jours), 2012 (45 jours), 1961 (44 jours), 1960 (42 jours), 1983 (42 jours), 2005 (42 jours), 1906 (41 jours), 1915 (41 jours) et 1991 (40 jours) (figure $2 b$ ). Ces évènements de très forte intensité apparaissent en hiver (préférentiellement lors du mois de février) et au cours de l'été (juin et août). Ils sont aussi fréquents dans la première moitié du $\mathrm{XX}^{\mathrm{e}}$ siècle que dans la seconde. La tendance quinquennale permet de distinguer plusieurs périodes de persistance de la sécheresse atmosphérique au cours du siècle (figure $2 \mathrm{~b}$ ). Par rapport à la valeur séculaire moyenne (13 jours), la décennie 1920-1930, les périodes 1940-1960 et 1988-1995 sont touchées par une succession d'évènements de plus forte intensité. La période récente 2010-2019 est une période moins touchée par la sécheresse atmosphérique malgré l'évènement de très forte intensité de 2012.

Les années 1945 et 1949 (Pardé, 1946 ; Raymon Rousset, 1946) et 1992 (Mérillon et Scherer, 1993) sont documentées dans la littérature scientifique sur des secteurs régionalement proches du bassin du Gardon. Des sources telles que les bases de données 
publiques EM-DAT (www.emdat.be/) et EDII (Blauhut et al., 2016) recensant les pertes humaines et économiques causées par les sécheresses, ont été mobilisées pour compléter les écrits scientifiques sur l'historique des sécheresses dans notre secteur d'étude (figure 2b). Elles recensent à partir de rapports d'instituts nationaux ou à travers les archives médiatiques, des impacts en lien avec les épisodes de sécheresses. Ces bases de données ne sont pas exhaustives, en particulier pour les périodes anciennes. L'absence d'une date dans ces bases de données ne remet pas en question la validité de l'évènement. Les années 1982, 1991 et 1997 sont recensées pour le secteur sud-est de la France dans la base de données EM-DAT. Les années 1991-1992, 2005-2006, 2011-2012 par EDII dans le Gard ou dans des départements à proximité (Lozère, Hérault). Enfin, les archives du département $\mathrm{du}$ Gard, disponibles sur le portail national des archives françaises (francearchives.fr/fr/) permettent de valider l'évènement 1906. Le document fait mention de calamités agricoles de type sécheresse cette année. Les années 1908, 1915, 1928, 1934, 1960 et 1961 n'ont pu être confirmées à partir des sources accessibles librement. Si des calamités agricoles sont identifiées ces années dans le document d'administration générale du département (1761-1961), l'origine sécheresse n'a pu être identifiée précisément.

\subsubsection{Probabilités d'occurrence des LMM (1 $\mathrm{mm}$ ) entre 1900 et 2019 en fonction de leur intensité au cours d'une année}

La loi Log-Normale a été déterminée comme la plus adaptée pour modéliser la fonction de probabilité des distributions des LMM de chaque mois de l'année (tableau 2). La loi Log-Normale, aussi appelé loi de Galton, noté $\log -\mathrm{N}(\mu, \sigma)$ est défini par deux paramètres : $\mu$ le mode et $\sigma$ la dispersion. Sa densité de probabilité est définie par l'équation suivante :

$$
f y(x ; \mu,, 6)=\frac{1}{x 6 \sqrt{2 \pi}} \exp \left(-\frac{(\ln x-\mu)^{2}}{26^{2}}\right)=\frac{1}{x} f x(\ln (x) ; \mu, 6)
$$

Les tests de Shapiro valident la normalité des résidus des lois log Normale utilisées à l'exception du mois de novembre (tableau 2). Un examen plus détaillé des résidus de ce mois montre la présence d'un second mode pour les intensités d'environ 20 jours. La présence de ce mode n'a pas pu être expliquée. L'examen graphique ne remet cependant pas en cause la forme générale de la distribution.

A intensité égale, les mois d'hiver et d'été ont une probabilité plus grande de générer des LMM intenses par rapport aux mois de printemps et d'automne, généralement plus pluvieux en climat méditerranéen (figure 4).
Figure 4. Fonctions de répartitions des longueurs maximales mensuelles (LMM) du sous-échantillon utilisé pour décrire la variabilité temporelle entre 1900 et 2019 des sécheresses atmosphériques (seuil de $1 \mathrm{~mm}$ ). Une fonction de répartition est représentée pour chaque mois de l'année. Distribution functions of the maximum monthly lengths (MML) of the subsample used to describe the temporal variability between 1900 and 2019 of atmospheric droughts $11 \mathrm{~mm}$ threshold). One distribution function is shown for each month of the year.

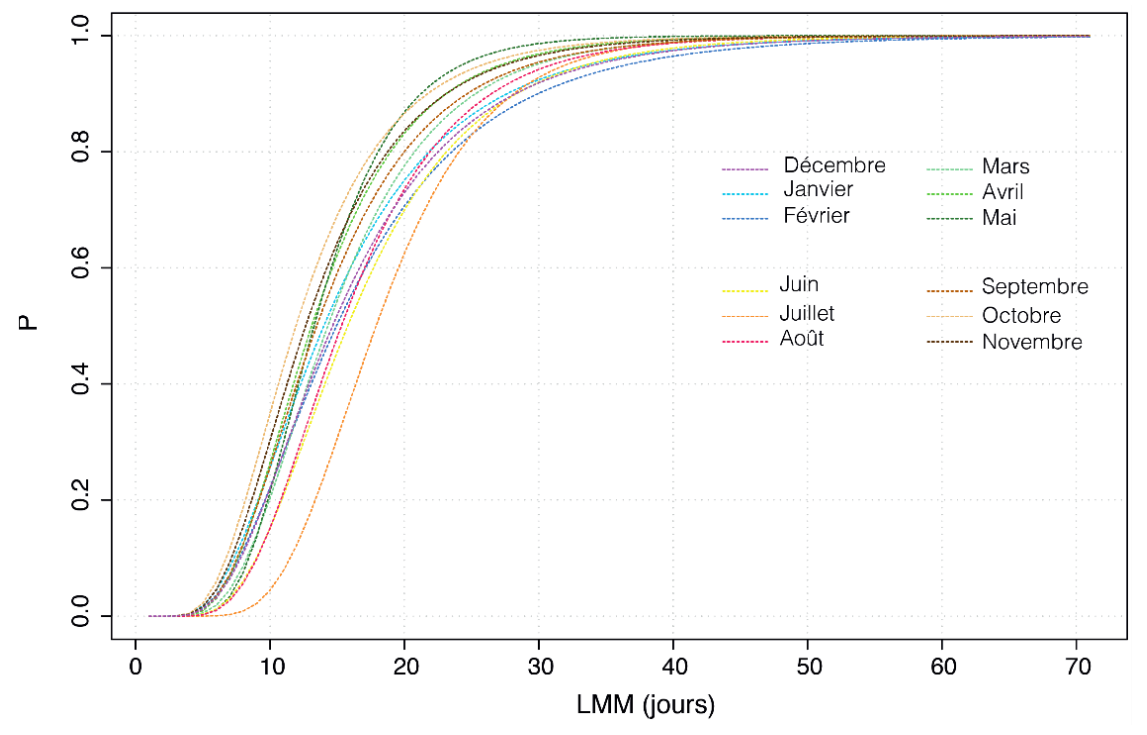

$P_{\text {août }}(L M M \geq 15)=0,45$. Juin est le mois d'été ayant la probabilité la plus importante de générer des évènements de très forte intensité $\mathrm{P}_{\text {juin }}(\mathrm{LMM} \geq 50)=$ 0,$005 ; \mathrm{P}_{\text {juillet }}(\mathrm{LMM} \geq 50)=0,001$ et $\mathrm{P}_{\text {août }}(\mathrm{LMM} \geq 50)$ $=0,002$. 
Cependant en hiver, les mois de décembre, janvier et février ont une plus grande probabilité de générer des évènements de très forte intensité par rapport aux mois d'été : $\mathrm{P}_{\text {décembre }}(\mathrm{LMM} \geq 50)=0,008$ ; $\mathrm{P}_{\text {janvier }}(\mathrm{LMM} \geq 50)=0,008$ et $\mathrm{P}_{\text {février }}(\mathrm{LMM} \geq 50)=$ $0,012)$. Les probabilités d'apparition d'évènements de moyenne intensité $P_{\text {décembre }}(\mathrm{LMM} \geq 30)=0,07$; $P_{\text {janvier }}(L M M \geq 30)=0,06$ et $P_{\text {février }}(L M M \geq 30)=0,08$ sont relativement proches de celles des mois d'été $\left(\mathrm{P}_{\text {juin }}(\mathrm{LMM} \geq 30)=0,07 ; \mathrm{P}_{\text {juillet }}(\mathrm{LMM} \geq 30)=0,06\right.$ et $\mathrm{P}_{\text {août }}(\mathrm{LMM} \geq 30)=0,04$. Elles sont cependant plus fortes en hiver pour les petites intensités : $\mathrm{P}_{\text {décembre }}(\mathrm{LMM} \geq 15)=0,43 ; \mathrm{P}_{\text {janvier }}(\mathrm{LMM} \geq 15)=$ 0,39 et $\mathrm{P}_{\text {février }}(\mathrm{LMM} \geq 15)=0,44$.

\subsection{Effet de la définition du jour sec sur la distribution statistique des LMM (2000-2019)}

\subsubsection{Probabilité d'occurrence des LMM en fonction du seuil (1 mm, ETP, ETR) sur l'ensemble de la période 2000-2019}

Sur la période de mesure du satellite, 44 stations sont disponibles pour calculer les séquences sèches entre 2000 et 2019 (figure 2a). Comme pour les distributions de l'échantillon séculaire des LMM (seuil $1 \mathrm{~mm}$ ), les fonctions de répartition pour les seuils $1 \mathrm{~mm}$, ETP et ETR entre 2000 et 2019 sont bien ajustées avec une loi Log-Normale (tableau 3). Le choix de la définition du jour sec n'implique pas de modification de la loi statistique. On distingue une fonction de répartition pour les LMM ETP qui se démarque des LMM $1 \mathrm{~mm}$ et LMM ETR (figure 5a). Un seuil d'ETP implique une probabilité d'occurrence d'évènement plus forte à intensité égale pour des évènements de faible ([0-15[ jours), moyenne ([15-30[ jours), forte ([30-40[ jours) et très forte intensité ([40-70] jours). Pour les évènements ayant une probabilité inférieure à 0,01 (évènement centennal), l'intensité pour le seuil 1 mm est supérieure à 40 jours, 44 jours avec le seuil d'ETR et 64 jours avec le seuil d'ETP (figure 5a). Un seuil d'ETP augmente de 0,04 la probabilité d'occurrence d'une LMM de 45 jours (maximum mesuré) calculée à partir d'un seuil de $1 \mathrm{~mm}$. Des LMM de plus de 70 jours peuvent être observées avec un seuil d'ETP contre 40 pour un seuil $1 \mathrm{~mm}$ et un seuil d'ETR.

Tableau 2. Paramètres des lois Log-Normales retenus pour les distributions des LMM de chaque mois du souséchantillon retenu entre 1900 et $2019: \mu$ la valeur modale de la distribution, $\sigma$ la dispersion de la distribution (plus $\sigma$ est grand, plus les queues de distribution sont longues) et $\mathrm{R}$ les indicateurs relatifs aux contrôles de la normalité des résidus d'ajustement de la loi. Les paramètres des lois retenus sont validés à partir d'un intervalle de confiance de 0,05. Parameters of the Log-Normal distributions selected for the MMLs distributions of each month of the retained subsample between 1900 and 2019: $\mu$ the modal value of the distribution, $\sigma$ the dispersion of the distribution (higher is $\sigma$ and longer is the tail of the distribution) and $R$ the indicators related to the controls of residual normality fitting. The parameters of the selected laws are validated with a confidence interval of 0.05 .

\begin{tabular}{|c|c|c|c|c|c|}
\hline $\begin{array}{c}\text { Distributions 1 mm } \\
(\mathbf{1 9 0 0 - 2 0 1 9 )}\end{array}$ & $\boldsymbol{\mu}$ & $\boldsymbol{\sigma}$ & \multicolumn{3}{|c|}{$\mathbf{R}$} \\
\cline { 4 - 6 } & & & Skewness & Kurtosis & Shapiro \\
\hline Janvier & $13,00 \pm 0,05$ & $0,56 \pm 0,06$ & 0,00 & 2,55 & 0,75 \\
\hline Février & $13,94 \pm 0,05$ & $0,57 \pm 0,06$ & 0,27 & 2,54 & 0,08 \\
\hline Mars & $13,30 \pm 0,04$ & $0,47 \pm 0,06$ & 0,15 & 2,81 & 0,10 \\
\hline Avril & $12,10 \pm 0,04$ & $0,47 \pm 0,06$ & $-0,09$ & 3,11 & 0,58 \\
\hline Mai & $12,27 \pm 0,03$ & $0,39 \pm 0,06$ & 0,08 & 3,22 & 0,46 \\
\hline Juin & $14,78 \pm 0,04$ & $0,48 \pm 0,06$ & 0,10 & 2,98 & 0,5 \\
\hline Juillet & $16,89 \pm 0,03$ & $0,37 \pm 0,06$ & $-0,21$ & 2,75 & 0,72 \\
\hline Août & $14,31 \pm 0,04$ & $0,45 \pm 0,06$ & 0,18 & 2,68 & 0,33 \\
\hline Septembre & $12,47 \pm 0,04$ & $0,50 \pm 0,06$ & $-0,11$ & 2,49 & 0,56 \\
\hline Octobre & $10,92 \pm 0,04$ & $0,50 \pm 0,06$ & 0,04 & 2,96 & 0,47 \\
\hline Novembre & $11,65 \pm 0,04$ & $0,50 \pm 0,06$ & $-0,13$ & 2,21 & 0,02 \\
\hline Décembre & $13,65 \pm 0,04$ & $0,54 \pm 0,06$ & $-0,05$ & 2,74 & 0,92 \\
\hline
\end{tabular}


Les fonctions de répartition pour le seuil ETR et celui d'1 $\mathrm{mm}$ sont relativement similaires (figure 5a). La fonction de répartition du seuil ETR montre à probabilité égale une intensité plus importante pour les longueurs faibles :
$\mathrm{P}_{1 \mathrm{~mm}}(\mathrm{LMM} \geq 15)=0,375 ; \quad \mathrm{P}_{\mathrm{ETR}}(\mathrm{LMM} \geq 15)=$ 0,444), moyennes : $\mathrm{P}_{1 \mathrm{~mm}}(\mathrm{LMM} \geq 30)=0,042$; $\mathrm{P}_{\mathrm{ETR}}(\mathrm{LMM} \geq 30)=0,061$ et les fortes : $\mathrm{P}_{1 \mathrm{~mm}}(\mathrm{LMM} \geq 40)=0,010 ; \operatorname{PeTR}(\mathrm{LMM} \geq 40)=0,016$ par rapport au seuil de $1 \mathrm{~mm}$.

Tableau 3. Paramètres des lois Log-Normales retenus pour les $L M M$ en fonction des différentes définitions du jour sec ( $1 \mathrm{~mm}, \mathrm{ETP}, \mathrm{ETR}$ ) pour les 44 stations disponibles entre 2000 et 2019. Avec $\mu$ le mode de la distribution, $\sigma$ la dispersion de la distribution (plus $\sigma$ est grand, plus les queues de distribution sont longues) et $R$ les indicateurs relatifs aux contrôles de la normalité des résidus d'ajustement de la loi. Les paramètres des lois retenus sont validés à partir d'un intervalle de confiance de 0,05. Parameters of the Log-Normal distributions retained for the MMLs according to the different definitions of dry days (1mm, ETP, ETR) for the 44 stations available between 2000 and 2019. With $\mu$ the mode of the distribution, $\sigma$ the dispersion of the distribution (higher is $\sigma$ and longer is the tail of the distribution) and $R$ the indicators related to the controls of residual normality fitting. The parameters of the selected laws are validated with a confidence interval of 0.05 .

\begin{tabular}{|c|c|c|c|c|c|}
\hline Distributions 2000-2019 & \multirow{2}{*}{$\boldsymbol{*}$} & \multicolumn{3}{|c|}{$\mathbf{R}$} \\
\cline { 4 - 7 } & & & Skewness & Kurtosis & Shapiro \\
\hline LMM 1 mm & $12,82 \pm 0,03$ & $0,49 \pm 0,04$ & -0.29 & 3,11 & 0,06 \\
\hline LMM ETR & $13,99 \pm 0,03$ & $0,49 \pm 0,04$ & $-0,26$ & 2,68 & 0,08 \\
\hline LMM ETP & $17,12 \pm 0,03$ & $0,56 \pm 0,04$ & $-0,21$ & 2,78 & 0,44 \\
\hline
\end{tabular}

Figure 5. Fonctions de répartition des LMM calculées à partir d'un seuil de 1 $\mathrm{mm}$, ETP et ETR entre 2000 et 2019 (a) et comparaisons pour chaque mois de l'année des distributions des différences de moyennes spatiales entre 2000 et 2019 pour les LMM $1 \mathrm{~mm}$ avec LMM ETP et les LMM $1 \mathrm{~mm}$ avec LMM ETR (b). La significativité des écarts des distributions de moyennes est vérifiée par un test de Wilcoxon. La $P$-value du test est indiquée sur le graphique lorsque des écarts n'ont pas été identifiés significatifs.

Distribution functions of LMMs calculated from a threshold of $1 \mathrm{~mm}$, ETP and ETR (a) and comparison for each month of the year of the distributions of spatial mean differences between 2000 and 2019 for $1 \mathrm{~mm}$ LMMs with ETP LMMs and $1 \mathrm{~mm}$ LMMs with ETR LMMs (b). The significance of the differences of the means between distributions is checked by a Wilcoxon test. The Pvalue of the test is indicated on the graph when differences have not been identified as significant. a.

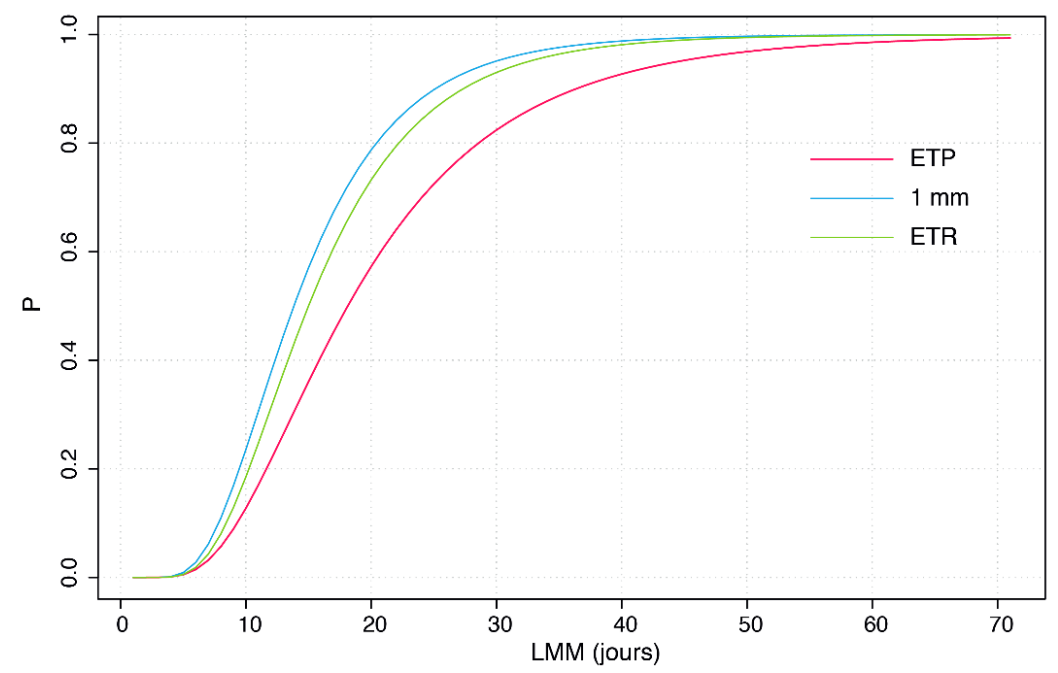

b.

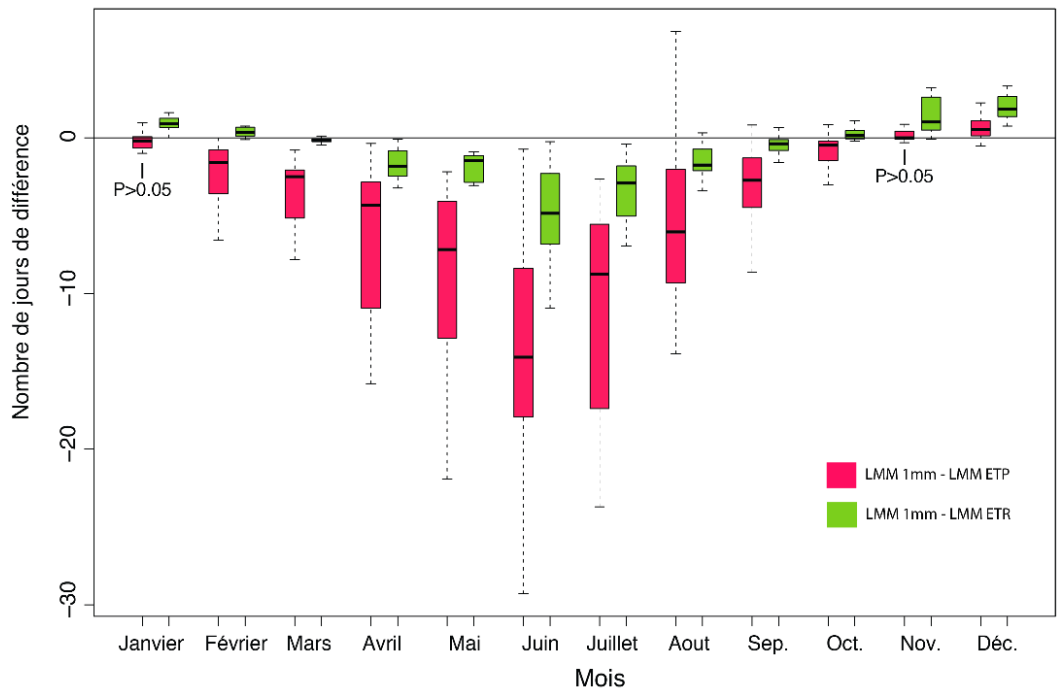




\subsubsection{Variation saisonnière des $L M M$ en fonction du seuil retenu (1 $\mathrm{mm}$, ETP, ETR)}

Le nombre d'individus par mois (20) est trop faible pour ajuster une loi statistique à partir des moyennes des intensités des LMM du secteur. De ce fait, nous limitons cette analyse aux différences de moyennes entre les LMM calculées à partir du seuil $1 \mathrm{~mm}$ et celles produites à partir des seuils d'ETP et d'ETR sur les 44 stations du secteur mesurant les précipitations entre 2000 et 2019 (figure 5b). Les écarts de moyennes dont la significativité est déterminée à partir d'un test de Wilcoxon sont plus importants avec un seuil d'ETP (entre 0 et -15 jours environ) qu'avec un seuil d'ETR (entre 2 et -7 jours environ). Ils sont majoritairement négatifs ( $98 \%$ pour l'ETP et $92 \%$ pour l'ETR) à l'exception de janvier $(0,75$ jour pour la valeur médiane), février $(0,38$ jour $)$, octobre $(0,15$ jour), novembre $(0,65)$, décembre $(1,70$ jours $)$ pour le seuil ETR. C'est en juin que les écarts négatifs avec 1'ETR ( -5 jours pour la valeur médiane) et l'ETP (-15 jours pour la valeur médiane) sont les plus importants. Les écarts sont très faibles et non significatifs pour les mois de janvier et novembre avec l'ETP. Les écarts sont significatifs quel que soit le mois pour l'ETR. A partir des valeurs moyennes du secteur, la détermination des PU avec un seuil d'ETP ou d'ETR montre une sousestimation de l'intensité des LMM avec un seuil 1 $\mathrm{mm}$ durant la période printanière et estivale (d'avril à août). On remarque aussi une légère surestimation des LMM en janvier, février, octobre, novembre et décembre avec un seuil $1 \mathrm{~mm}$ par rapport au seuil d'ETR.

\subsubsection{Distribution spatiale des paramètres de forme ( $\mu$ et $\sigma$ ) des lois de distribution des LMM retenues pour chaque station en fonction du seuil (1 mm, ETP, ETR)}

L'utilisation d'une loi Log-Normale est toujours pertinente pour déterminer la probabilité d'occurrence des LMM sur chacune des 44 stations du secteur. Deux paramètres définissent la forme de la distribution des lois Log-Normale : $\mu$ fixe le mode et $\sigma$ la dispersion de la distribution. Plus $\sigma$ est grand et plus les queues de distribution sont longues. Les tests de Shapiro-Wilk ne valident pas la normalité des résidus sur 8 stations pour l'ETP, 7 pour l'ETR et 11 pour $1 \mathrm{~mm}$. L'examen détaillé de ces résidus a montré la présence d'un deuxième mode pour les valeurs (ETP, ETR, $1 \mathrm{~mm}$ ) dans la gamme des intensités moyennes ([15-30] jours). Il est probablement lié à un effet de taille des échantillons, plus petite que pour ceux utilisés dans le cadre de l'analyse effectuée entre 1900 et 2019. Pour ces stations problématiques, nous avons recherché si les résidus de ces modes pouvaient avoir un effet levier sur la droite d'Henry des résidus de l'ajustement de la loi Log-Normale. Dans ce cas de figure, les résidus peuvent remettre en cause la qualité d'ajustement de la loi et induire des biais dans l'estimation des paramètres. Deux stations sur 8 sont impactées par des résidus problématiques pour l'ETP, 4 sur 7 pour l'ETR et 4 sur 11 pour $1 \mathrm{~mm}$ (figure 6). Entre 2 et 4 stations par carte restent problématiques pour la détermination correcte des paramètres des lois.

Le choix du seuil implique une augmentation de $\mu$ ( $\mu$ ETP compris entre 12 et 18 jours; $\mu$ ETR entre 9 et 15 jours et $\mu 1 \mathrm{~mm}$ entre 9 et 15 jours) mais n'induit pas de modifications importantes de sa répartition spatiale (figure 6). L'ensemble du bassin est touché par des évènements d'intensité de moyennes ampleurs ( $\mu \mathrm{ETP}$ compris entre 12 et 15 jours ; $\mu \mathrm{ETR}$ entre 9 et 13 jours et $\mu 1 \mathrm{~mm}$ entre 9 et 12 jours) relativement plus faible que les secteurs au nord de celui-ci, à l'exception de la partie amont du sousbassin du Gardon d'Ales (sous-bassin en amont de la station de la Grand- Combes), du sous-bassin de la Salindrenque (sous-bassin en amont de la station Lassale) de plus forte intensité (figures 1 et 6). L'intensité moyenne est la plus forte en aval du bassin du Gardon ( $\mu_{\text {ETP }}, \mu_{\text {ETR, }} \mu_{1 \mathrm{~mm}}$ entre 15 et 19 jours environ).

Comme pour le paramètre $\mu$, le choix du seuil implique une augmentation de $\sigma$ (бETP compris entre 0,578 et 0,707 ; бETR compris entre 0,519 et 0,642 ; $\sigma 1 \mathrm{~mm}$ compris entre 0,460 et 0,585$)$. Cependant, le seuil à un impact beaucoup plus marqué sur la distribution spatiale du paramètre $\sigma$ (figure 6). La partie la plus en aval du bassin est concernée par des fortes valeurs pour le seuil ETP et $1 \mathrm{~mm}$ (бETP compris entre 0,578 et 0,$707 ; \sigma_{1 \mathrm{~mm}}$ compris entre $0,460$ et 0,585$)$. Le seuil $1 \mathrm{~mm}$ implique des valeurs de $\sigma$ relativement faibles sur la grande majorité du bassin et donc peu de variabilité spatiale contrairement aux autres seuils. Le seuil d'ETR 
permet quant à lui de mettre en évidence une opposition entre secteurs amont (Cévennes) concernés par des valeurs relativement plus fortes de $\sigma$ (comprises entre 0,561 et 0,642 ) que la plaine (comprises entre 0,519 et 0,558). A l'inverse, le seuil d'ETP implique des valeurs de sigma plus faibles en amont (comprises entre 0,578 et 0,633) que sur le reste du bassin, à l'exception du secteur amont du sous-bassin de la Salindrenques (sousbassin en amont de la station Lassale). On ne retrouve pas de gradient altitudinal, contrairement aux cumuls annuels moyens de précipitation, pour les évènements de moyenne intensité. Une légère relation statistiquement significative $(\mathrm{P}<0,05)$ d'après un test de Bravais-Pearson est identifiée entre la valeur du paramètre $\sigma E T R$ et l'altitude $\left(\mathrm{R}^{2}=\right.$ 0,34). L'altitude semble être un facteur qui accentue la probabilité d'occurrence des évènements les plus intenses pour les LMM calculées avec un seuil ETR. Elle n'est cependant pas suffisante pour bien expliquer la géographie de $\sigma$.

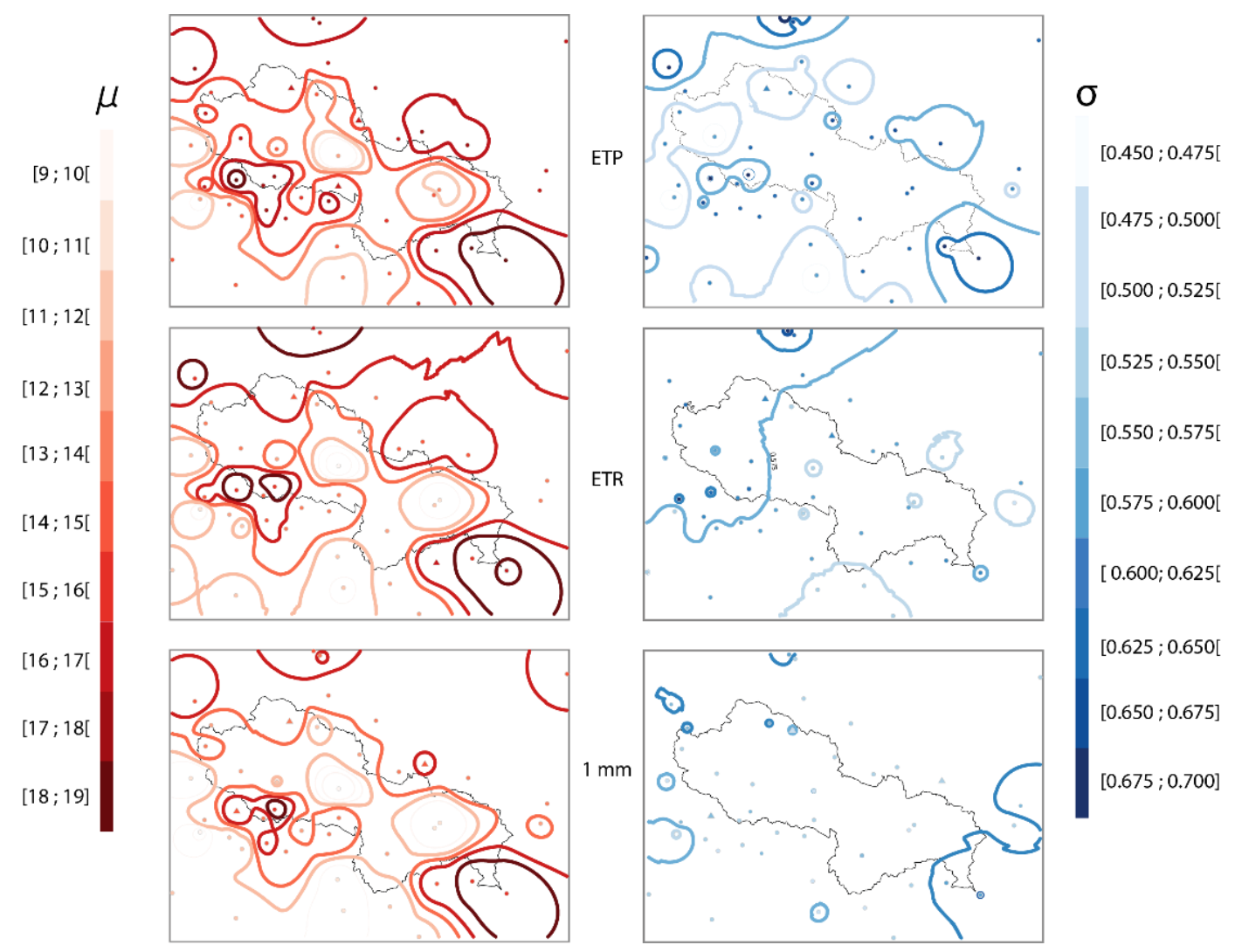

Figure 6. Cartographie des paramètres de distribution des lois Log-Normales. $\mu$ correspond à la longueur modale de la distribution des séquences sèches et $\sigma$ au paramètre de dispersion de la loi statistique log normale retenue. Plus la valeur du paramètre $\sigma$ est grande, plus les queues de distribution sont longues et plus les probabilités d'occurrence des évènements de forte et très forte intensité sont élevées. Les cartes sont reproduites pour les trois définitions du jour sec retenues (ETP, ETR et $1 \mathrm{~mm}$ ). Une interpolation IDW a été utilisée pour mieux identifier les structures spatiales. Un paramètre de friction égale à 1,5 a été retenu pour mieux dégager les structures spatiales dominantes et limiter, sans pour autant faire disparaître l'impact des effets locaux de certaines stations. La qualité des interpolations a été contrôlée par validation croisée. Les isolignes de cette interpolation permettent de dégager la structure spatiale de chaque variable. Les stations dont l'estimation des paramètres de la loi pourrait être problématique sont figurées par un triangle. Mapping of the distribution parameters of Log-Normal distributions. $\mu$ corresponds to the average length of the distribution of dry sequences and $\sigma$ the dispersion parameter of the distribution. Higher are the parameter $\sigma$, longer the distribution tails are and higher the probability of occurrence of high and extreme intensity events. The maps are reproduced for the three dry day definitions used (PET, ET and $1 \mathrm{~mm}$ ). An IDW interpolation was used to better identify the spatial structures. A friction parameter equal to 1.5 was used to better identify the dominant spatial structures and to limit the impact of local effects of some stations without making them disappear. The quality of the interpolations was checked by cross-validation. The isolines of this interpolation improve the visibility of the spatial structure of each variable. Stations for which the estimation of the parameters of the law could be problematic are represented by a triangle. 


\section{Discussion}

La comparaison des estimations in situ et par satellite permet de vérifier la cohérence entre les deux méthodes mais ne renseigne pas sur la meilleure façon d'estimer l'ETP ou l'ETR. Si les comparaisons montrent une bonne similitude, celles avec l'ETR le sont un peu moins. Des comparaisons à partir d'autres stations in situ et d'autres approches méthodologiques de l'estimation de l'ETR doivent être mises en œuvre pour préciser la validité des mesures MODIS.

Nous n'avons pas détecté d'assèchement séculaire significatif à partir des LMM (seuil $1 \mathrm{~mm}$ ) sur le secteur du Gardon. Si la diminution, relativement faible, des pluies est constatée pour quelques mois de l'année dans le sud-est de la France (Norrant et Douguédroit, 2004), elle n'est pas reliée à une tendance d'intensification des LMM. Ces résultats rejoignent ceux de Raymond et Ullmann (2021) qui n’ont pas identifié une augmentation générale statistiquement significative de la longueur moyenne des séquences sèches dans le sud-est de la France entre 1950 et 2019. Dans ce même travail, ce constat est également fait pour les séquences sèches les plus longues. Cependant, quelques secteurs locaux sont identifiés comme significatifs par les auteurs. A proximité de notre zone d'étude (piémont de la façade sud-est du Massif central), durant l'hiver, une hausse statistiquement significative de l'augmentation de la longueur moyenne est détectée. Plus située au niveau de la vallée du Rhône, toujours en hiver, une augmentation significative des séquences sèches les plus longues est également détectée. Dans notre cas, nous n'avons pas pu confirmer ces tendances entre 1900 et 2019 à partir des LMM (seuil $1 \mathrm{~mm}$ ) de notre sous-échantillon. A l'exception du mois de janvier durant lequel la $\mathrm{P}$-value ne permet pas d'affirmer avec certitude la non significativité (faiblesse de la significativité au seuil de 0,05 du test de Mann-Kendall).

La confirmation des années touchées par des évènements très intenses à partir d'impact de sécheresse identifié par d'autres sources est un résultat encourageant afin de valider l'intérêt des LMM pour le suivi des sécheresses sur le bassin du Gardon. On observe parfois un décalage d'un an entre l'évènement de très forte intensité identifiée et les impacts. Ce décalage entre l'évènement et l'impact est typique de la lenteur du processus de sécheresse (Senaut, 2015 ; Wilhite, 2000). L'extension spatiale d'une sécheresse est généralement de grande envergure (d'une centaine à plusieurs milliers de kilomètres) (Senaut, 2015 ; Van Loon, 2015). Valider ces mesures avec des évènements régionaux, pas strictement localisés sur notre secteur d'étude, reste acceptable. Certains évènements de la première moitié $\mathrm{du} \mathrm{XX}^{\mathrm{e}}$ siècle n'ont pas pu être validés faute de sources, comme 1928 et 1934, touchées l'une et l'autre par un évènement très intense. La sécheresse 1906, pourtant de moindre intensité, est à l'inverse référencée. Des recherches en archives plus approfondies sont nécessaires pour confirmer ou infirmer ces évènements. Il est possible que leurs impacts aient été plus faibles. Un biais de souséchantillonnage est aussi possible.

Confirmer des évènements passés à partir d'indicateurs reste un exercice difficile. Il n'existe pas encore de réel consensus sur le terme sécheresse, à cause de la diversité des indicateurs et des impacts (Charre, 1977 ; Dorize, 1990 ; Mishra et Singh, 2010 ; Martin et al., 2020). Parfois, l'argument de période chaude rentre en compte pour désigner la sécheresse. L'une est souvent associée à l'autre, mais la chaleur amplifie surtout l'intensité de la sécheresse par augmentation de l'évapotranspiration (Vicente-Serrano et al., 2020 ; Vicente-Serrano et al., 2014). Le facteur déterminant reste l'impluviosité. Quelquefois, la sécheresse est observée à partir d'impacts sociétaux marquants (pertes agricoles, famines, feux de forêt, etc.). La présence d'impacts est un marqueur d'une sécheresse. Une concordance entre un aléa de forte intensité et des impacts permet de conforter notre confiance sur la pertinence de l'indicateur. Cependant, il ne permet pas à lui seul de dicter avec précision la gravité des impacts, car elle est dépendante de la vulnérabilité de chaque territoire (non constant dans le temps et l'espace) (Stahl et al., 2016 ; Wilhite et al., 2007 ; Ault et al., 2016 ; Birkmann et al., 2007 ; Blauhut et al., 2016).

La longueur des séquences sèches les plus longues et la saisonnalité des intensités (seuil $1 \mathrm{~mm}$ ) observées sur le secteur du bassin du Gardon rejoignent les observations déjà produites dans le 
sud-est de la France à partir d'autres jeux de données (Douguédroit, 1980, 1990 ; Raymond et Ullmann, 2021). Les fonctions de répartition pour les différents mois de l'année des LMM (1 mm) entre 1900 et 2019 montrent des intensités moyennes plus élevées pour les mois d'été et d'hiver. Les longs épisodes estivaux signalent une absence d'épisodes orageux pouvant ralentir le tarissement des cours d'eau. Les LMM d'hiver sont à probabilité égale encore plus intenses que celles d'été (parmi les 16 épisodes les plus intenses du siècle 10 sont hivernaux). Celles-ci sont problématiques pour la recharge des grands aquifères.

Un jour sec fondé sur l'ETR augmente la probabilité d'occurrence des faibles et moyennes LMM par rapport au seuil de $1 \mathrm{~mm}$. L'utilisation d'un seuil ETR donne à probabilité égale des intensités relativement similaires à un seuil $1 \mathrm{~mm}$. Les LMM des mois de printemps et début d'été sont plus intenses avec un seuil ETR. Le mois de juin est particulièrement sous-estimé avec le seuil $1 \mathrm{~mm}$. Or, il s'agit du mois d'été qui a la plus grande probabilité de générer des épisodes de très fortes intensités avec un seuil de $1 \mathrm{~mm}$. Des évènements printaniers intenses peuvent limiter la période de recharge des aquifères. Pour des secteurs où les aquifères sont peu capacitifs (amont du bassin du Gardon par exemple), leur alimentation pendant le printemps est cruciale pour maintenir un débit de rivière suffisant pour l'irrigation alimentée par les écoulements de surface. Ces évènements sont mieux identifiables avec un seuil d'ETR. Il est également possible que l'intensité des évènements hivernaux soit légèrement surestimée par le seuil de $1 \mathrm{~mm}$. De plus, les différences entre ETR et $1 \mathrm{~mm}$ pourraient être encore plus fortes s'il existe bien un biais d'estimation des plus faibles et plus fortes valeurs d'ETR par MODIS pour les mois d'hiver (surestimation des faibles valeurs par rapport aux mesures de la tour de flux de turbulence) et de fin de printemps (sous-estimation des pics annuels d'ETR par rapport à la tour de flux de turbulence Fluxnet).

L'utilisation d'un seuil d'ETP semble surestimer, quel que soit la probabilité d'occurrence, l'intensité des LMM par rapport à un seuil d'ETR et de $1 \mathrm{~mm}$. S'il permet de prendre en compte l'effet saisonnier de l'évapotranspiration, contrairement au seuil de $1 \mathrm{~mm}$, celui-ci surestime fortement à probabilité d'occurrence égale l'intensité d'une séquence sèche (pour un seuil 1 mm entre +50 et $+90 \%$ pour les faibles intensités, entre +15 et $+60 \%$ pour les moyennes intensités, entre +4 et $+14 \%$ pour les fortes intensités). Selon nous, le seuil d'ETR permet de mieux considérer le stress de la végétation du bassin. En cas d'indisponibilité d'information pour l'ETR, le seuil de $1 \mathrm{~mm}$ nous semble plus réaliste car celui-ci exagère beaucoup moins l'intensité des séquences sèches.

L'effet du seuil sur la distribution spatiale des paramètres des distributions Log-Normales des stations est faible pour le paramètre $\mu$ (intensités ayant une probabilité d'occurrence de 0,5). L'intensité augmente en fonction du seuil mais la distribution spatiale reste relativement identique. A l'inverse, on observe beaucoup plus de variabilité avec la distribution spatiale du paramètre $\sigma$ lorsqu'on change la définition du jour sec. Par exemple, à partir du seuil d'ETR, on constate que la tête de bassin du Gardon est concernée par des valeurs plus élevées de $\sigma$ par rapport à la partie plaine. La probabilité des très fortes valeurs d'intensités est donc plus élevée en amont qu'en aval. Ce qui peut sembler contre-intuitif de prime à bord si l'on cherche à faire le lien avec la distribution spatiale des cumuls annuels climatiques de précipitation.

Ainsi, l'effet de la végétation et/ou la réserve utile joue un rôle non négligeable sur la distribution spatiale et la saisonnalité de l'intensité des LMM. Quel que soit le seuil, parmi les secteurs les plus touchés (valeurs de $\sigma$ et $\mu$ ), il existe un risque important pour le sous-bassin de la Salindrenque (686 ha de cultures correspondant à $15 \%$ des surfaces cultivées du secteur cévenol) et l'aval du bassin (quasi exclusivement cultivé). Douguédroit (1983) a montré que la quantité d'eau tombée est faiblement corrélée avec la longueur des séquences sèches dans le sud-est de la France. Nos résultats semblent rejoindre ce constat. Il est probable que les évènements intenses soient davantage liés à des phases anticycloniques de vaste extension spatiale. 


\section{Conclusion}

Une analyse locale des séquences sèches a permis de caractériser l'aléa sécheresse atmosphérique sur le bassin du Gardon, avec une observation de l'évolution des sécheresses atmosphériques entre 1900 et 2019 à partir d'un seuil de pluie couramment utilisé : $1 \mathrm{~mm}$. Aucune tendance significative n'a été détectée. A l'exception de 1908, 1915, 1928, 1934, 1961 et 1962, nous n'avons pas pu confirmer des impacts témoignant de sécheresse à proximité et dans le secteur du bassin du Gardon. A notre connaissance, une analyse de la sécheresse atmosphérique à partir de données locales n'a pas encore été proposée pour ce bassin.

Ensuite, cette étude a permis de vérifier la pertinence d'utilisation de l'imagerie satellite MODIS pour la mesure de l'ETR et l'ETP sur le bassin du Gardon. Elle s'est avérée de bonne qualité à une échelle hebdomadaire pour le suivi de l'ETP et de qualité correcte pour l'ETR à une échelle saisonnière. Ces données pourraient être utiles dans des secteurs où la couverture spatiale des mesures in situ pour l'ETP et l'ETR (beaucoup plus rarement mesurée) est insuffisante. La plus grande incertitude sur les mesures d'ETR appelle à réaliser des analyses plus approfondies (nature des biais et validité des mesures quotidiennes) à partir d'autres stations et dans d'autres secteurs méditerranéens.

Puis, nous avons soulevé l'importance de l'effet du choix de la définition du jour sec sur l'intensité des séquences sèches. Le seuil ETP surestime considérablement l'intensité des séquences sèches à probabilité d'occurrence égale alors qu'elles sont relativement proches du seuil $1 \mathrm{~mm}$ pour les intensités de faibles, moyennes, fortes et quasiment identiques pour les plus fortes. Au risque de surestimer l'intensité de la sécheresse atmosphérique sur le bassin du Gardon, il nous semble plus judicieux d'utiliser un seuil $1 \mathrm{~mm}$ plutôt que celui de l'ETP à défaut d'avoir une mesure de l'ETR. Le seuil ETR a aussi montré que le mois de juin (plus grande probabilité de produire des évènements très intenses en été) est probablement sous-estimé avec un seuil $1 \mathrm{~mm}$ et les mois de décembre et janvier sont légèrement surestimés. La détermination des séquences sèches à partir de l'ETR permet un suivi plus réaliste des évènements impactant pour la végétation d'un bassin. Il pourrait être plus efficace pour établir le lien entre sécheresse hydrologique et édaphique. Des études complémentaires sont à mener à partir d'autres jeux de données (rendement agricole, humidité des sols et débit des rivières par exemple) pour le vérifier.

Enfin, l'analyse de la distribution spatiale des paramètres de formes des lois de distribution des longueurs des séquences sèches (2000-2019) a permis de montrer l'impact du choix du seuil du jour sec sur la distribution spatiale de la probabilité d'occurrence des séquences sèches les plus longues. Le seuil ETR a permis de montrer que le secteur cévenol à une probabilité plus élevée d'être touché par les évènements de sécheresse atmosphérique de très forte intensité que la partie plaine du bassin. Cette structure spatiale n'a pas été retrouvée avec les autres seuils. Nous avons aussi détecté que le sous-bassin de la Salindrenque en Cévennes et la partie la plus en aval du bassin étaient les plus à risque. La probabilité d'occurrence d'un évènement intense est plus forte dans ces deux secteurs à l'activité agricole importante.

L'analyse des facteurs explicatifs de la genèse des évènements les plus longs mérite d'être approfondie. Si nous avons constaté une faible relation avec l'altitude pour le seuil ETR, nous soupçonnons que des forçages anticycloniques longs permettent de mieux expliquer le maintien des longs épisodes sans pluie. Plusieurs auteurs ont montré un lien entre situation de blocage atmosphérique et période impluvieuse en secteur méditerranéen. L'impact de processus atmosphérique tel que l'Oscillation NordAtlantique (ONA) est connue pour induire un basculement récurrent entre période humide et sèche en secteur méditerranéen à une échelle régionale (Trambley et Hertig, 2018 ; Raymond et al., 2018).

Afin d'améliorer notre compréhension sur le maintien de longues périodes sans pluie dans le secteur du Gardon, une analyse corrélative entre l'intensité des évènements et la pression atmosphérique doit être entreprise. 
Remerciements: Les données de stations météorologiques utilisées dans cet article ont été obtenues dans le cadre d'une convention recherche entre le laboratoire ESPACE et Météo France. D'autres données ont pu être téléchargées gratuitement depuis le site internet de la Nasa (modis.gsfc.nasa.gov/data/), EM-Dat (www.emdat.be/), de l'European Drougth Center (europeandroughtcentre.co $\underline{\mathrm{m}}$ ) et de FLUXNET (fluxnet.org/data/fluxnet2015dataset/). Cette recherche a été possible grâce au financement d'une bourse doctorale attribué par la Fédération de Recherche Agorantic (agorantic.univ -avignon.fr/). Nous remercions les deux relecteurs anonymes pour leurs retours critiques sur le manuscrit de cet article qui ont permis l'amélioration de ce travail. De vifs remerciements sont également adressés aux professeurs D. Blanke et P. Martin pour leurs conseils et aides durant la réalisation de ce travail.

\section{Références}

Aguilera-Klink F., Perez-Moriana E. et Sanchez-García J., 2000. The social construction of scarcity. The case of water in Tenerife (Canary Islands). Ecological Economics, 34(2), 233 -245. https://doi.org/10.1016/S0921-8009(00)00160-9

Arnell N. W., 2003. Effects of IPCC SRES* emissions scenarios on river runoff: a global perspective. Hydrology and Earth System Sciences, 7(5), 619-641. https://doi.org/10.5194/hess-7-6192003

Ault T. R., Justin S. M.,Cook B. I., Smerdon J. E., 2016. Relative impacts of mitigation, temperature, and precipitation on 21st-century megadrought risk in the American Southwest. Science Advances, 2(10). https://www.science.org/doi/abs/10.1126/sciadv.1600873

Birkmann J., 2007. Risk and vulnerability indicators at different scales: Applicability, usefulness and policy implications. Environmental hazards, 7(1), 20-31, 10.1016/j.envhaz.2007.04.002

Blauhut V., Stahl K., Stagge J. H., Tallaksen L. M., De Stefano L. et Vogt, J., 2016. Estimating drought risk across Europe from reported drought impacts, drought indices, and vulnerability factors. Hydrology and Earth System Sciences, 20(7), 2779-2800. https://doi.org/10.5194/hess-20-27792016

Burke E. J. et Brown S. J., 2008. Evaluating uncertainties in the projection of future drought. Journal of Hydrometeorology, 9(2), 292-299. https://doi.org/10.1175/2007JHM929.1

Calianno M., Milano M. et Reynard E., 2018. Monitoring water use regimes and density in a tourist mountain territory. Water Resources Management, 32(8), 2783-2799. https://doi.org/10.1007/s11269-018-1958-9

Caloiero T., Coscarelli R., Ferrari E. et Sirangelo B., 2015.
Analysis of dry spells in southern Italy (Calabria). Water, 7(12), 3009-3023. https://doi.org/10.3390/w7063009

Carrega P., 1988. L'évapotranspiration potentielle et réelle dans le Midi méditerranéen. Son originalité par rapport au reste de le France. Méditerranée, 66(4), 3-8.

Charre J., 1977. A propos de sécheresse. Revue de géographie de Lyon, 52(2), 215-226.

Cleveland W. S., 1981. LOWESS: A program for smoothing scatterplots by robust locally weighted regression. The American Statistician, 35(1), 54. http://dx.doi.org/10.2307/2683591

Dai A., 2011. Drought under global warming: a review. Wiley Interdisciplinary Reviews: Climate Change, 2(1), 45-65. https://doi.org/10.1002/wcc.81

De Marsily G., 2010. L'eau et ses grands enjeux au XXI siècle : effet sur la zone aride. Sécheresse, 21(1), 12-21.

Deni S. M. et Jemain A. A., 2009. Mixed log series geometric distribution for sequences of dry days. Atmospheric Research, 92(2), 236-243. https://doi.org/10.1016/j.atmosres.2008.10.032

Dorize L., 1990. La sécheresse: en quête d'une définition. Sécheresse, 1(1), 1-10.

Douguedroit A., 1980. La sécheresse estivale dans la région Provence Alpes Côte d'Azur. Méditerranée, 39(2), 13-21.

Douguedroit A., 1983. Un siècle de sécheresse estivale à Marseille. Hommes et Terres du Nord, 3(1), 34-38.

Douguedroit A., 1987. The variations of dry spells in Marseilles from 1865 to 1984. Journal of Climatology, 7(6), 541-551. https://doi.org/10.1002/joc.3370070603

Douguedroit A., 1990. Spécificité et variations de la sécheresse le long du littoral méditerranéen français. Revue de géographie de Lyon, 65(2), 123-128.

Établissement Public Territorial de Bassin Gardons, 2018. Plan de gestion de la ressource en eau, 122 pages.

Dracup J. A., Lee K. S. et Paulson E. G., 1980. On the definition of droughts. Water Resources Research, 16(2), 297 -302. https://doi.org/10.1029/WR016i002p00297

Ducharne A., 2002. Projet GICC-Seine : questions relatives à l'ETP, 10 pages.

Folton N., Martin É., Arnaud P. et Tolsa, M., 2020. Cinquante ans de processus hydrologiques observés dans des petits bassins versants méditerranéens : vers une raréfaction de la ressource en eau? La Houille Blanche, 106(5), 17-27. https://doi.org/10.1051/lhb/2020048

Garcia C. C. et Martin-Vide J., 1993. Analyse par la chaîne de Markov de la sécheresse dans le sud-est de l'Espagne. Sécheresse, 4(2), 123-129.

Giorgi F., 2006. Climate change hot-spots. Geophysical Research Letters, 33(8), 4. http://doi.wiley.com/10.1029/2006GL025734

Guermazi E., Milano M., Reynard E., Zairi, M., 2019. Impact of climate change and anthropogenic pressure on the groundwater resources in arid environment. Mitigation and Adaptation Strategies for Global Change, 24, 73-92. doi.org/10.1007/s11027-018-9797-9 
Hamed K. H. et Ramachandra Rao A., 1998. A modified MannKendall trend test for autocorrelated data. Journal of Hydrology, 204(1 -4), 182-196. https://doi.org/10.1016/S0022-1694(97)00125-X

Jaeger W. K., Plantinga A. J., Chang H., Dello K., Grant G., Hulse D., Mcdonnell J. J., Lancaster S., Moradkhani H., Morzillo A. T., Mote P., Nolin A., Santelmann M. et Wu J., 2013. Toward a formal definition of water scarcity in naturalhuman systems: Opinion. Water Resources Research, 49(7), 4506-4517. https://doi.org/10.1002/wrcr.20249

Kamar K. et Rao T.V., 2004. Dry and wet spells at Campina Granade. Revista Brasileira de Meteorologia, 20(1), 71-74.

Karavitis C. A., Tsesmelis D. E., Skondras N. A., Stamatakos D., Alexandris S., Fassouli V., Vasilakou C. G., Oikonomou P. D., Gregoric G., Grigg N. S. et Vlachos E. C., 2014. Linking drought characteristics to impacts on a spatial and temporal scale. Water Policy, 16(6), 1172-1197. https://doi.org/10.2166/wp.2014.205

Kendall M. G. et Gibbons J. D., 1990. Rank correlation methods (5th ed.). London: New York, NY: E. Arnold. Oxford University Press, 21.

Lana X., Burgueño A., 1998. Probabilities of repeated long dry episodes based on the Poisson distribution. An example for Catalonia (NE Spain). Theor. Appl. Climatol., 60, 111-120. https://doi.org/10.1007/s007040050037

Lana X., Burgueño A., Martinez M. D. et Serra, C., 2006. Statistical distributions and sampling strategies for the analysis of extreme dry spells in Catalonia (NE Spain). Journal of Hydrology, 324(1-4), 94-114. https://doi.org/10.1016/j.jhydrol.2005.09.013

Lana X., Martinez M. D., Burgueño A. et Serra, C., 2008. Return period maps of dry spells for Catalonia (northeastern Spain) based on the Weibull distribution. Hydrological Sciences Journal, 53(1), 48-64. https://doi.org/10.1623/hysj.53.1.48

Lecarpentier C., 1975. L'évapotranspiration potentielle et ses implications géographiques. Annales de Géographie, 84(464), 385-414.

Mann H. B., 1945. Nonparametric tests against trend. Econometrica, 13(3), 245. https://doi.org/10.2307/1907187

Martin P., Di Costanzo H. et Canovas I., 2020. Aridité et sécheresse: heuristique d'une modélisation parétienne et bayésienne de la tension sur la ressource en eau dans le sudest de la France. Journal International Sciences et Technique de l'Eau et de l'Environnement, 2(21).

McKee T. B., Doesken N. J. et Kleist J., 1993. The relationship of drought frequency and duration to time scales. 8th Conference on Applied Climatology, Anaheim, 17-22 January 1993, 179-184.

Merillon Y. et Scherer J. C., 1993. La sécheresse de 1992 : Bilan - Conséquences - Enseignements. La Houille Blanche, 79(8), 559-570. https://doi.org/10.1051/lhb/1993047

Milano M., Ruelland D., Dezetter A., Fabre J., Ardoin-Bardin S., Servat, E., 2013. Modeling the current and future capacity of water resources to meet water demands in the Ebro basin. Journal of Hydrology, 500, 114-126. doi.org/10.1016/j.jhydrol.2013.07.010
Mishra A. K. et Singh V. P., 2010. A review of drought concepts. Journal of Hydrology, 391(1-2), 202-216. https://doi.org/10.1016/j.jhydrol.2010.07.012

Monteith J. L., 1965. Evaporation and environment. Symposia of the Society for Experimental Biology, 19, 205-234.

Moreno Á., Garcia-Haro F., Martinez B. et Gilabert M., 2014. Noise reduction and gap filling of fAPAR time series using an adapted local regression Filter. Remote Sensing, 6(9), 82388260. https://doi.org/10.3390/rs6098238

Mounier J., 1977. Aspects et fréquences de la sécheresse en Bretagne : essai de définition de la sécheresse en Europe océanique. Revue de géographie de Lyon, 52(2), 161-176.

Norrant C. et Douguedroit A., 2004. Tendances des précipitations mensuelles et quotidiennes dans le sud-est méditerranéen français (1950-51 / 1999-2000). Annales de l'Association Internationale de Climatologie, 1, 45-64. https://doi.org/10.4267/climatologie.987

Norrant C. et Douguedroit A., 2006. Monthly and daily precipitation trends in the Mediterranean (1950-2000). Theoretical and Applied Climatology, 83(1-4), 89-106. https://doi.org/10.1007/s00704-005-0163-y

Palmer W. C., 1965. Meteorological Drought. Research paper of U.S. Department of Commerce, 45, 65.

Parde M., 1946. La sécheresse des années 1941-1946. Annales de l'université de Grenoble, 22, 99-103.

Pastorello G., 2020. The FLUXNET2015 dataset and the ONEFlux processing pipeline for eddy covariance data. Sci. Data, 7(225), 27. https://doi.org/10.1038/s41597-020-0534-3

Peiris T., 2011. Critical Evaluation of Dry Spell Research. International Journal of Basic \& Applied Sciences, 11(06), 153-160.

Perzyna G., 1994. Spatial and temporal characteristics of maximum dry spells in Southern Norway. International Journal of Climatology, 14(8), 895-909. https://doi.org/10.1002/joc.3370140806

Raymond F. et Ullmann A., 2021. Variabilité spatiotemporelle des séquences sèches en France depuis 1950. Cybergeo, 27. https://doi.org/10.4000/cybergeo.37260

Raymond F., Ullmann A., Camberlin P., Drobinski P. et Smith, C. C., 2016. Extreme dry spell detection and climatology over the Mediterranean Basin during the wet season: dry spell over the mediterranean basin. Geophysical Research Letters, 43(13), 7196-7204. https://doi.org/10.1002/2016GL069758

Raymond F., Ullmann A., Camberlin P., Oueslati B. et Drobinski P., 2018. Atmospheric conditions and weather regimes associated with extreme winter dry spells over the Mediterranean basin. Climate Dynamics, 50(11-12), 44374453. https://doi.org/10.1007/s00382-017-3884-6

Riaux J., Barbier R., Barreteau O., 2009. Construire et argumenter des enjeux de vulnérabilité en comité sécheresse. Risques et environnement : Recherches interdisciplinaires sur la vulnérabilité des sociétés, L'Harmattan, 75-87. 
Rigby R., Stasinopoulos M., Heller G., Bastiani, F. D., 2017. Distributions for modelling location, scale and shape: Using GAMLSS in R. Technical paper, 378. https://doi.org/10.1007/s00382-017-3884-6

Rivoire P., Tramblay Y., Neppel L., Hertig E. et VicenteSerrano S. M., 2019. Impact of the dry day definition on Mediterranean extreme dry spells analysis. Natural Hazards and Earth System Sciences, 19, 1629-1638. https://doi.org/10.5194/nhess-2019-31

Rousset R., 1946. A propos de quatre années de sécheresse : 1942-1945. Revue de Géographie Alpine, 34(2), 313-318.

Running S. W., Mu Q., Zhao M. et Moreno, A., 2019. User's guide MODIS global terrestrial evapotranspiration (ET) product (MOD16A2/A3 and year-end gap-filled $M O D 16 A 2 G F / A 3 G F)$. MODIS Land Team, 40.

Şenaut Z., 2015. Applied drought modeling, prediction, and mitigation. Amsterdam: Elsevier, 474 pages.

Shapiro S. S. et Wilk M. B., 1965. An analysis of variance test for normality (complete samples). Biometrika, 52(3-4), 591611. https://doi.org/10.2307/2333709

Soubeyroux J., Vidal J.-P., Najac J., Kitova N., Blanchard M., Dandin P., Martin E., Pages C. et Habets F., 2011. Impact du changement climatique en France sur la sécheresse et l'eau du sol. Rapport final du projet ClimSec, 72 pages.

Sousa P. M., Trigo R. M., Aizpurua P., Nieto R., Gimeno L. et Garcia-Herrera R., 2011. Trends and extremes of drought indices throughout the 20th century in the Mediterranean. Natural Hazards and Earth System Sciences, 11(1), 33-51. https://doi.org/10.5194/nhess-11-33-2011

Spinoni J., Naumann G. et Vogt J. V., 2017. Pan-European seasonal trends and recent changes of drought frequency and severity. Global and Planetary Change, 148, 113-130. https://doi.org/10.5194/nhess-11-33-2011

Stagge J. H., Kingston D. G., Tallaksen L. M. et Hannah, D. M., 2017. Observed drought indices show increasing divergence across Europe. Scientific Reports, 7(1), 10. https://doi.org/10.1038/s41598-017-14283-2

Stahl K., Kohn I., Blauhut V., Urquijo J., De Stefano L., Acácio V., Dias S., Stagge J. H., Tallaksen L. M., Kampragou E., Van Loon A. F., Barker L. J., Melsen L. A., Bifulco C., Musolino D., de Carli A., Massarutto A., Assimacopoulos D., Van Lanen H. A. J., 2016. Impacts of European drought events: insights from an international database of text-based reports. Natural hazards earth system Science, 16, 801-819. https://doi.org/10.5194/nhess-16-801-2016.

Stasinopoulos D. M. et Rigby R. A., 2007. Generalized Additive Models for Location Scale and Shape (GAMLSS) in R. Journal of Statistical Software, 7(7), 1-46. https://doi.org/10.18637/jss.v023.i07

Thornthwaite C. W., 1948. An approach toward a rational classification of climate. Geographical Review, 38(1), 55-94.

Tramblay Y., Hertig E., 2018. Modelling extreme dry spells in the Mediterranean region in connection with atmospheric circulation. Atmospheric Research, 202, 40-48. https://doi.org/10.1016/j.atmosres.2017.11.015

Tramblay Y., Koutroulis A., Samaniego L., Vicente-Serrano S. M., Volaire F., Boone A., Le Page M., Llasat M. C., Albergel C., Burak S., Cailleret M., Kalin K. C., Davi H., Dupuy J.-L., Greve P., Grillakis M., Hanich L., Jarlan L., Martin-Stpaul N., Martínez-Vilalta J., Mouillot F., Pulido-Velazquez D., Quintana-Seguí P., Renard D., Turco M., Türkeş M., Trigo R., Vidal J.-P., Vilagrosa A., Zribi M. et Polcher J., 2020. Challenges for drought assessment in the Mediterranean region under future climate scenarios. Earth-Science Reviews, 210, 24. https://doi.org/10.1016/j.earscirev.2020.103348

Van Der Schrier G., Briffa K. R., Jones P. D. et Osborn T. J., 2006. Summer Moisture Variability across Europe. Journal of Climate, 19(12), 2818-2834. https://doi.org/10.1175/JCLI3734.1

Van Loon A. F., 2015. Hydrological drought explained: Hydrological drought explained. Wiley Interdisciplinary Reviews: Water, 2(4), 359-392. https://doi.org/10.1002/wat2.1085

Van Loon A. F., Gleeson T., Clark J., Van Dijk A. I. J. M., Stahl K., Hannaford J., Di Baldassarre G., Teuling A. J., Tallaksen L. M., Uijlenhoet R., Hannah D. M., Sheffield J., Svoboda M., Verbeiren B., Wagener T., Rangecroft S., Wanders N. et Van Lanen H. A. J., 2016a. Drought in the Anthropocene. Nature Geoscience, 9(2), 89-91. https://doi.org/10.1038/ngeo2646

Van Loon A. F., Stahl K., Di Baldassarre G., Clark J., Rangecroft S., Wanders N., Gleeson T., Van Dijk A. I. J. M., Tallaksen L. M., Hannaford J., Uijlenhoet R., Teuling A. J., Hannah D. M., Sheffield J., Svoboda M., Verbeiren B., Wagener T. et Van Lanen H. A. J., 2016b. Drought in a humanmodified world: reframing drought definitions, understanding, and analysis approaches. Hydrology and Earth System Sciences, 20(9), 3631-3650. https://doi.org/10.5194/hess-20-3631-2016

Vannier O. et Braud I., 2012. Calcul d'une évapotranspiration de référence spatialisée pour la modélisation hydrologique à partir des données de la réanalyse SAFRAN de Météo-France. IRSTEA, 24 pages.

Vicente-Serrano S. M. et Begueria-Portugues S., 2003. Estimating extreme dry-spell risk in the middle Ebro valley (northeastern Spain): a comparative analysis of partial duration series with a general Pareto distribution and annual maxima series with a Gumbel distribution: dry spell risk in ne spain. International Journal of Climatology, 23(9), 11031118. https://doi.org/10.1002/joc.934

Vicente-Serrano S. M., Lopez-Moreno J.-I., Begueria S., Lorenzo-Lacruz J., Sanchez-Lorenzo A., Garcia-Ruiz J. M., Azorin-Molina C., Moran-Tejeda E., Revuelto J., Trigo R., Coelho F. et Espejo F., 2014. Evidence of increasing drought severity caused by temperature rise in southern Europe. Environmental Research Letters, 9(4), 23. http://dx.doi.org/10.1088/1748-9326/9/4/044001

Vicente-Serrano S. M., Mcvicar T. R., Miralles D. G., Yang Y. et Tomas-Burguera M., 2020. Unraveling the influence of atmospheric evaporative demand on drought and its response to climate change. WIREs Climate Change, 11(2), 31. https://doi.org/10.1002/wcc.632 
Wilcoxon, F., 1945. Individual Comparisons by Ranking Methods. Biometrics Bulletin, 1(6), 80. https://doi.org/10.2307/3001968

Wilhite D. A., 2000. Chapter 1 - Drought as a natural hazard: concepts and definitions. Drought Mitigation Center Faculty Publications, 69, $22 \mathrm{p}$.

Wilhite D. A. et Glantz M. H., 1985. Understanding the Drought Phenomenon: The Role of Definitions. Water International, 10(3), 111-120.

Wilhite D. A., Svoboda M. D. et Hayes M. J., 2007. Understanding the complex impacts of drought: A key to enhancing drought mitigation and preparedness. Water Resources Management, 21(5), 763-774. https://doi.org/10.1007/s11269-006-9076-5

Zhang K., Kimball J. S. et Running, S. W., 2016. A review of remote sensing based actual evapotranspiration estimation: A review of remote sensing evapotranspiration. Wiley Interdisciplinary Reviews: Water, 3(6), 834-853. https://doi.org/10.1002/wat2.1168

Citation de l'article : Di Costanzo H., 2021. Probabilités d'occurrence des séquences sèches les plus longues sur le bassin versant du Gardon (sud-est de la France) - Quels impacts de la définition du jour sec sur les distributions statistiques des séquences sèches les plus longues en climat méditerranéen ? Climatologie, 18, 6 . 